\title{
LOS HOTSPOTS: EXPANSIÓN DE LAS TAREAS OPERATIVAS Y COOPERACIÓN MULTILATERAL DE LAS AGENCIAS EUROPEAS FRONTEX, EASO Y EUROPOL
}

\author{
DAVID FERNÁNDEZ ROJO' \\ Universidad de Deusto \\ davidfrojo@deusto.es
}

\begin{abstract}
Cómo citar/Citation
Fernández Rojo, D. (2018).

Los hotspots: expansión de las tareas operativas y cooperación multilateral de las agencias europeas Frontex, Easo y Europol.

Revista de Derecho Comunitario Europeo, 61, 1013-1056. doi: https://doi.org/10.18042/cepc/rdce.61.06
\end{abstract}

\section{Resumen}

La aproximación de los hotspots es una de las principales medidas adoptadas por parte de la UE para hacer frente a la «crisis de los refugiados». Esta aproximación requiere que, conforme a un marco operativo común, las agencias europeas Frontex, Easo y Europol refuercen su cooperación multilateral y asistan a aquellos Estados miembros sujetos a presiones excepcionales de flujos migratorios mixtos en sus sistemas nacionales de registro, recepción y asilo. Dada la reciente introducción de esta aproximación y la falta de transparencia acerca de las concretas tareas conferidas a las agencias, este artículo analiza en qué medida los hotspots refuerzan la cooperación multilateral y expanden las tareas operativas de Frontex, Easo y Europol sobre el terreno. Tras el estudio del funcionamiento de los hotspots, se concluye que las tareas operativas que desempeñan en la práctica las agencias exceden tanto la mera asistencia

1 Investigador en formación, Universidad de Deusto. Beneficiario del Programa de Formación de Personal Investigador no Doctor del Departamento de Educación, Política Lingüística y Cultura del Gobierno Vasco. Agradezco a los evaluadores anónimos los comentarios realizados sobre el presente trabajo. 
técnica requerida en sus mandatos legales como la doctrina de no delegación del TJUE. Es necesario, por tanto, que el poder legislativo de la Unión adopte un instrumento vinculante en el que se detalle y delimite la cooperación, tareas y responsabilidad de las agencias en los hotspots.

\title{
Palabras clave
}

Hotspots; Frontex; Easo; Europol; cooperación multilateral.

\section{THE HOTSPOTS: THE REINFORCEMENT OF THE OPERATIONAL TASKS AND MULTILATERAL COOPERATION OF THE EUROPEAN AGENCIES FRONTEX, EASO AND EUROPOL}

\begin{abstract}
The hotspot approach was one of the key measures adopted by the EU in the aftermath of the "refugee crisis». This approach requires the European agencies Frontex, Easo and Europol to strengthen their multilateral cooperation and operationally assist those Member States subject to exceptional migratory pressure in their national registration, reception and asylum systems. Given the recent introduction of the hotspots and the lack of transparency regarding the specific competences that the agencies may develop on the ground, this article analyzes to what extent the hotspot approach reinforces the multilateral cooperation and the operational tasks of Frontex, Easo and Europol. After examining the functioning of the hotspots, it is concluded that the operational tasks conducted in practice by these agencies exceed their legal mandates and the CJEU's non-delegation doctrine. Therefore, the legislative power of the EU should adopt a binding instrument in which the cooperation, competences and responsibility of the agencies in the hotspots is detailed and delimited.
\end{abstract}

\section{Keywords}

Hotspots; Frontex; Easo; Europol; multilateral cooperation.

\section{LES HOTSPOTS: RENFORCEMENT DES TÂCHES OPÉRATIONNELLES ET DE LA COOPÉRATION MULTILATÉRALE DES AGENCES EUROPÉENNES FRONTEX, EASO ET EUROPOL}

\section{Résumé}

L'approche hotspots est l'une des principales mesures prises par l'UE pour faire face à la "crise des réfugiés». L'approche exige que, dans un cadre opérationnel commun, les agences européennes Frontex, Easo et Europol renforcent leur coopération 
multilatérale et aident les États membres soumis à des pressions exceptionnelles de flux migratoires mixtes dans leurs systèmes nationaux d'enregistrement, d'accueil et d'asile. Compte tenu de l'introduction récente de cette approche et du manque de transparence à propos des compétences spécifiques conférées aux agences, cet article analyse dans quelle mesure les hotspots renforcent la coopération multilatérale et étendent les tâches opérationnelles de Frontex, d'Easo et d'Europol sur le terrain. Après avoir étudié le fonctionnement des hotspots, il est conclu que les tâches opérationnelles que les agences exécutent dans la pratique vont au-delà de la simple assistance technique requise dans leurs mandats légaux, ainsi que de la doctrine de non-délégation de la CJUE. Il est donc nécessaire que le législateur de l'Union adopte un instrument contraignant qui détaille et délimite la coopération, les compétences et la responsabilité des agences dans les hotspots.

\section{Mots clés}

Hotspots; Frontex; Easo; Europol; coopération multilatérale. 


\section{SUMARIO}

I. INTRODUCCIÓN. II. LA COOPERACIÓN MULTILATERAL ENTRE FRONTEX, EASO Y EUROPOL. III. LA APROXIMACIÓN DE LOS HOTSPOTS: UNA COOPERACIÓN REFORZADA, MULTILATERAL Y OPERATIVA ENTRE FRONTEX, EASO Y EUROPOL: 1. Definiendo el enfoque de los hotspots. 2. Funcionamiento y división de tareas entre Frontex, Easo y Europol en los hotspots: 2. 1. La asistencia operativa de Frontex en el registro, la identificación y el retorno de los migrantes irregulares. 2.2. La asistencia de Easo en el registro y el examen de las solicitudes de asilo. 2.3. La asistencia de Europol en la prevención y lucha contra el tráfico ilegal de migrantes y la trata de seres humanos. IV. EL IMPACTO Y LA COMPATIBILIDAD DE LOS HOTSPOTS CON LOS REGLAMENTOS DE FRONTEX, EASO Y EUROPOL Y CON LA DOCTRINA DEL TJUE DE NO DELEGACIÓN: 1. El limitado impacto inicial de los hotspots. 2. Los mandatos legales de Frontex, Easo y Europol y las tareas operativas desarrolladas en la práctica por las agencias en los hotspots. 3. La doctrina del TJUE de no delegación como límite a la expansión de la cooperación multilateral entre Frontex, Easo y Europol. V. CONCLUSIÓN. BIBLIOGRAFía.

\section{INTRODUCCIÓN}

La «crisis de los refugiados» ha puesto de relieve la existencia de un déficit en la aplicación efectiva y uniforme por parte de los Estados miembros de las leyes y políticas de la Unión Europea (UE) en materia de migración, asilo y gestión de fronteras ${ }^{2}$. El refuerzo del mandato de la Oficina Europea de Policía (Europol) ${ }^{3}$, la creación de una Guardia Europea de Fronteras y Costas $(\mathrm{GEFC})^{4}$ y la propuesta de transformar la actual Oficina Europea de

2 Del Valle Gálvez (2016: 759-777); Den Heijer et al. (2016: 607-642); Triandafyllidou y Mantanika (2017: 7-38); Thym (2016: 1556).

3 Reglamento (UE) 2016/794 del Parlamento Europeo y del Consejo de 11 de mayo de 2016 relativo a la Agencia de la Unión Europea para la Cooperación Policial (Europol) (DO L 135 de 24.05.2016). Véase Blasi Casagran (2016: 202-221).

4 Reglamento (UE) 2016/1624 del Parlamento Europeo y del Consejo de 14 de septiembre de 2016 sobre la Guardia Europea de Fronteras y Costas (DO L 251 de 16.09.2016). Véanse Carrera y Den Hertog (2016: 1-19); Campesi (2018: 1-32); 
Apoyo al Asilo (Easo) en una Agencia Europea de Asilo (AEA) ${ }^{5}$ durante 2016 responden a la necesidad de garantizar la implementación de las medidas operativas adoptadas en el marco del Espacio de Libertad, Seguridad y Justicia (ELSJ) de la UE. Tanto la GEFC como la futura AEA están llamadas a asistir operativamente sobre el terreno a aquellas autoridades nacionales sujetas a presiones extraordinarias en sus sistemas de registro, recepción y asilo, así como a garantizar respectivamente el funcionamiento del espacio Schengen y el Sistema Europeo Común de Asilo (SECA). Europol, en lo que a migración, asilo y gestión de fronteras respecta, apoya a los Estados miembros en sus investigaciones nacionales en materia de tráfico ilegal de migrantes y trata de seres humanos.

Si bien el nuevo nombre con el que se ha bautizado a la anteriormente conocida como Agencia Europea para la Gestión de la Cooperación Operativa en las Fronteras Exteriores (Frontex) parece sugerir que la UE cuenta ya con un verdadero Cuerpo Europeo de Guardias de Fronteras al que han sido conferidas funciones exclusivas y ejecutivas en el control de las fronteras, el Reglamento 2016/1624 se limita meramente a reforzar las tareas delegadas inicialmente a Frontex. Tal y como señala De Bruycker (2016: 559-569), la GEFC es una ficción legal conformada por la suma de la nueva agencia y las autoridades nacionales, que continuarán encargándose en último término de la gestión de sus fronteras exteriores. En la práctica, la GEFC sigue denominándose Frontex y este es el término empleado en este trabajo para referirse a la agencia.

No solo las tareas operativas conferidas a Frontex, Easo y Europol se han fortalecido (Scipioni, 2017: 1-17), sino que la cooperación multilateral entre estas agencias también se ha promovido tras la introducción de los hotspots ${ }^{6}$ por la Agenda Europea de la Migración en 2015. Si bien estas agencias ya venían cooperando tanto bilateral como multilateralmente, la «crisis de los refugiados» ha revelado la necesidad de desarrollar una cooperación multilateral sistemática y operativa en el territorio de aquellos Estados miembros más afectados por la llegada masiva de flujos migratorios mixtos. La efectiva cooperación entre Frontex, Easo y Europol es una prioridad en un ELSJ cada vez más integrado, el cual precisa de una Administración fuerte que implemente

Fernández Rojo (2017: 223-251); Ferraro y De Capitani (2016: 385-398); Pérez González (2016: 192-208); Rijpma (2016); Santos Vara (2018: 143-186); Soler García (2017: 1-44).

5 Propuesta de Reglamento del Parlamento Europeo y del Consejo relativo a la Agencia de Asilo de la Unión Europea y por el que se deroga el Reglamento (UE) 439/2010, $\operatorname{COM}(2016) 271$ final, 4.5.2016. Véase Tsourdi (2016: 997-1031).

6 En castellano "puntos críticos», aunque este trabajo emplea la terminología inglesa por ser más reconocible. 
de manera efectiva y uniforme la política migratoria, de asilo y de gestión fronteriza de la Unión.

Mientras que la doctrina se ha centrado mayoritariamente en estudiar las tareas conferidas a Frontex, Easo y Europol por separado, este artículo analiza en qué medida los hotspots refuerzan la cooperación multilateral y expanden las tareas operativas de estas tres agencias sobre el terreno - hasta la fecha y en un sentido más amplio solo Horii (2018: 1-27); Rodier (2017:1-22), y Basilien-Gainche (2017: 153-171)—. Dada la reciente creación de los hotspots, la falta de transparencia acerca de su funcionamiento y la inexistencia de un instrumento normativo que especifique las funciones operativas que desarrollan Frontex, Easo y Europol en los mismos, este trabajo examina las tareas conferidas a estas agencias y las actividades que llevan a cabo en la práctica en los hotspots. Para ello, la primera sección explora el concepto y la evolución de la cooperación multilateral entre Frontex, Easo y Europol (II). Seguidamente, se detalla el funcionamiento de los hotspots y las concretas funciones operativas que cada una de las agencias tiene encomendadas en los mismos (III). Por último, se analiza el impacto de los hotspots y, en concreto, la conformidad de la cooperación multilateral reforzada de Frontex, Easo y Europol tanto con sus respectivos mandatos legales como con la doctrina de no delegación del Tribunal de Justicia de la Unión Europea (TJUE) (IV). El estudio finaliza con un breve apartado de conclusiones (V).

\section{LA COOPERACIÓN MULTILATERAL ENTRE FRONTEX, EASO Y EUROPOL}

Frontex, Easo y Europol, cuyas tareas están estrechamente interrelacionadas, nunca se han limitado a coexistir, sino que desde su creación se ha fomentado su cooperación. El art. 13 del Reglamento 2007/2004, que creó Frontex, indicaba que «la Agencia podrá cooperar con Europol y con las organizaciones internacionales competentes en los ámbitos regulados por el presente Reglamento, en el marco de acuerdos de trabajo celebrados con dichos organismos, de acuerdo con las disposiciones pertinentes del Tratado y con las disposiciones sobre la competencia de dichos organismos» ${ }^{7}$. El art. 22.1 de la decisión del Consejo, de 6 de abril de 2009, por la que se introdujo Europol como agencia descentralizada señalaba que «en la medida en que ello sea útil

7 Reglamento (CE) 2007/2004 del Consejo de 26 de octubre de 2004 por el que se crea una Agencia Europea para la gestión de la cooperación operativa en las fronteras exteriores de los Estados miembros de la Unión Europea (DO L 349 de 25.11.2004). 
para el ejercicio de sus funciones, Europol podrá establecer y mantener relaciones de cooperación con las instituciones, las agencias y los organismos creados por los Tratados [...]» ${ }^{8}$. Por último, el art. 52 del Reglamento 439/2010 de Easo apunta que «la Oficina de Apoyo cooperará con los organismos de la Unión que desempeñen actividades relacionadas con su ámbito de competencia, y en particular con Frontex y la FRA»?

La cooperación exigida en su momento por los reglamentos de Frontex, Easo y Europol se ha materializado gradualmente tanto en comunicaciones informales entre las agencias (por ejemplo, reuniones o intercambio de buenas prácticas e información) como a través de relaciones más formales cuyo fin radica en desarrollar coherentemente las tareas operativas de las agencias y asistir con efectividad a los Estados miembros ${ }^{10}$. Esto es, la cooperación inicial entre Frontex, Easo y Europol ha evolucionado desde el mero intercambio de información y recursos a través de la firma de acuerdos bilaterales hasta la actual cooperación multilateral sobre el terreno, la realización de tareas y proyectos operativos conjuntos y el intercambio de recursos. El ámbito de estudio de este artículo se centra en analizar la cooperación multilateral operativa reforzada entre Frontex, Easo y Europol en los hotspots, lo que excluye cualquier otro tipo de cooperación bilateral, intercambio de información o cooperación que acontezca entre estas tres agencias.

La cooperación multilateral entre Frontex, Easo y Europol en los hotspots pretende hacer frente a la característica fragmentación, duplicación y superposición que surge entre agencias que desarrollan sus tareas en materias tan interrelacionadas como las incluidas en el ELSJ. Ante materias proclives al surgimiento de duplicaciones o superposiciones como son la migración, el asilo o la gestión de fronteras exteriores consideramos que existen tres opciones de actuación: no cooperar, cooperar o fusionar las agencias implicadas. Esta sección analiza estos tres escenarios con el objetivo de clarificar las razones que llevaron a la Comisión Europea a promover la cooperación multilateral operativa entre Frontex, Easo y Europol en los hotspots.

El mandato de Frontex radica fundamentalmente en apoyar a las autoridades nacionales para que gestionen eficazmente sus fronteras exteriores; el de

8 Decisión del Consejo, de 6 de abril de 2009, por la que se crea la Oficina Europea de Policía (Europol) (DO L 121 de 15.5.2009).

9 Reglamento (UE) n. ${ }^{\circ}$ 439/2010 del Parlamento Europeo y del Consejo de 19 de mayo de 2010 por el que se crea una Oficina Europea de Apoyo al Asilo (DO L 132 de 29.05.2010).

10 Argomaniz (2012: 72-94); Christiansen (2001: 747-769); Donaire Villa (2015: 3946); Hertog y Strob (2013: 373-388). 
Easo, en asistir a los Estados miembros en la aplicación eficaz y uniforme del SECA, y la misión de Europol se centra en apoyar a los Estados miembros en la lucha contra las formas de delincuencia que afecten a un interés común protegido por una política de la UE. Si bien los mandatos de Frontex, Easo y Europol parecen a priori claramente delimitados, estas agencias asisten a los Estados miembros en ámbitos como, por ejemplo, la llegada repentina y excepcional de flujos migratorios mixtos en la frontera exterior de un Estado miembro, en los que el mandato de las tres agencias se ve afectado. Ante esta situación, puede optarse por no promover ningún tipo de cooperación entre Frontex, Easo y Europol y que cada agencia asista por separado al Estado miembro que haya requerido su apoyo operativo. Aunque ante este supuesto de no cooperación Frontex, Easo y Europol serían plenamente independientes y responsables a la hora de desempeñar las tareas de apoyo que les han sido conferidas, la asistencia operativa resultante estaría sujeta a duplicaciones y superposiciones. Ello redundaría en una aproximación inconsistente e insuficiente ante una situación que requiere necesariamente de la cooperación entre las tres agencias.

El segundo escenario que se presenta para la UE y los Estados miembros ante situaciones que involucren simultáneamente los mandatos de Frontex, Easo y Europol es promover su cooperación. Pese a que aun cooperando puedan surgir superposiciones y duplicaciones entre las agencias, la fragmentación de acción ante un desafío común disminuirá significativamente. Ahora bien, ha de tenerse en cuenta que, en ausencia de un marco normativo que delimite los objetivos compartidos de estas agencias y que garantice la autonomía y caracteres distintivos de cada agencia, Frontex, Easo y Europol tenderán a competir entre ellas para lograr una mayor influencia e independencia sobre el ámbito material sobre el que ejercen sus funciones (Busuioc, 2016: 44). Hasta el momento, los reglamentos de Frontex, Easo y Europol vagamente regulan su cooperación y son las propias agencias las que en la práctica desarrollan y perfilan sus relaciones bilaterales y multilaterales (Weyemberg et al., 2014: 8-9).

El último escenario residiría en que el legislador europeo decidiese fusionar Frontex, Easo y Europol en una sola agencia ${ }^{11}$. A este respecto, el enfoque común para las agencias de la UE adoptado por la Comisión Europea, el Parlamento y el Consejo en 2012 estableció que la fusión de agencias debería ser una opción a considerar en aquellos supuestos en los que haya un riesgo de que sus tareas puedan superponerse, su actuación sea más eficiente tras integrarse en una estructura superior o se generen importantes sinergias operati-

11 Brady (2008: 103-109); Carrera et al. (2017: 56); Den Heijer et al. (2016: 607642); Guild (2016: 585-600); Goodwin-Gill (2015: 1-16). 
vas $^{12}$. Obviamente, el diseño de una única agencia eliminaría cualquier riesgo de solapamiento o duplicación, pero la singularidad y la naturaleza de cada agencia también desaparecería tras la fusión. En la práctica, la unión de agencias es un escenario no exento de dificultades debido al rechazo por parte de las propias agencias a su fusión, así como la cuestionable necesidad de integrar agencias con tareas cuya naturaleza y lógica difiere.

En el ELSJ se negociaron infructuosamente dos fusiones de agencias: el Instituto Europeo de la Igualdad de Género (EIGE) con la Agencia de los Derechos Fundamentales (FRA), y Europol con la Escuela Europea de Policía (Cepol). Respecto a la fusión de EIGE y FRA, la Comisión Europea evaluó en su propuesta de reglamento si las tareas del instituto deberían incluirse dentro del ámbito de la FRA. La Comisión argumentó que la creación de una agencia independiente estaba justificada debido al avanzado estado de desarrollo y la especificidad de la política de igualdad de género, que va más allá de la lucha contra la discriminación y el respeto de un derecho fundamental ${ }^{13}$.

Por otro lado, la reciente propuesta de la Comisión Europea de fusionar Europol y Cepol estuvo sujeta a un mayor escrutinio y debate público. Según la Comisión, su fusión "podría crear sinergias importantes y mejorar su eficiencia [...], ayudarían a identificar las necesidades de formación [...], se evitaría la duplicación de funciones de apoyo en ambas agencias y los ahorros resultantes podrían reasignarse e invertirse en funciones operativas y de formación básicas» ${ }^{14}$. Tanto Europol como Cepol mostraron su oposición a su fusión al estimar que tienen misiones, tareas y mandatos bien diferenciados y al entender que su cooperación nunca fue tan sobresaliente como para justificar la creación de una única agencia ${ }^{15}$. El Parlamento Europeo también

12 Joint Statement of the European Parliament, the Council of the EU and the European Commission on decentralized agencies, Annex: Common Approach, 19.07.2012, p. 3. Disponible en: https://bit.ly/2hvgKR9 (todas las fuentes electrónicas utilizadas han sido recuperadas el 23 de abril de 2018).

13 Propuesta de Reglamento del Parlamento Europeo y del Consejo por el que se crea un Instituto Europeo de la Igualdad de Género, COM(2005) 81 final, 08.03.2005, p. 4.

14 Propuesta de Reglamento del Parlamento Europeo y del Consejo relativo a la Agencia de la Unión Europea para la cooperación y la formación en funciones coercitivas (Europol), COM(2013) 173 final, 27.03.2013, p. 4. Véase Annexes to the Impact Assessment on merging the European Police College (Cepol) and the European Police Office (Europol) and implementing a European police training scheme for law enforcement officials, SWD(2013) 98 final, 27.03.2013.

15 CEPOL Position Paper, European Commission Package on the creation of a European Agency for Law Enforcement Cooperation and Training. Disponible en: https://bit.ly/ 2tRAkxV. 
rechazó la integración de Cepol en Europol argumentando que estas agencias perderían su autonomía y singularidad, y que las agencias ya llevaban a cabo sus tareas de manera efectiva y en estrecha cooperación ${ }^{16}$. Finalmente, la integración de Cepol en Europol no prosperó.

Desde la UE se ha optado por la opción de promover la cooperación entre las agencias que forman parte del ELSJ. Dada la proliferación de agencias en este espacio y el constante refuerzo de sus mandatos, su cooperación multilateral es una prioridad para la Unión (Pi Llorens, 2017: 77-117). En el ELSJ la cooperación multilateral entre las agencias se empezó a incentivar en el año 2006 a través de reuniones anuales de los directores de las agencias en las que se intercambian buenas prácticas y se determinan áreas comunes de trabajo ${ }^{17}$. Asimismo, en 2008 se recomendó a las agencias del ELSJ que mejoraran la complementariedad de sus actividades al preparar sus programas de trabajo anuales y que garantizaran un intercambio fluido de información ${ }^{18}$. No fue, sin embargo, hasta la entrada en vigor en 2009 del Tratado de Lisboa que la cooperación multilateral entre las agencias devino en un objetivo prioritario a alcanzar en el ELSJ.

El Programa de Estocolmo adoptado por el Consejo Europeo a finales de 2009, que concretó la agenda del ELSJ durante el periodo 2010-2014, expuso la necesidad de perfeccionar la coordinación interna para evitar duplicaciones y lograr una mayor coherencia entre las políticas, leyes y actuaciones de las agencias ${ }^{19}$. En este sentido, la Presidencia sueca en 2010 solicitó a las agencias que evaluasen su cooperación multilateral ${ }^{20}$. El informe adoptado subrayó que la interacción y las sinergias operativas entre las agencias del ELSJ debían aún reforzarse si se quería explotar al máximo todo el potencial de sus mandatos ${ }^{21}$.

Un segundo informe elaborado por las agencias del ELSJ durante el año 2010 para la Presidencia española también analizó su cooperación multilateral y advirtió que aún cabían opciones de mejora ${ }^{22}$. Específicamente, el informe recomendó el desarrollo de sinergias en el campo de la investigación y la

16 Parlamento Europeo, Civil Liberties MEPs discuss CEPOL and Europol merger, 07.05.2013. Disponible en: http://bit.ly/2B0s8P1.

17 Consejo de la Unión Europea, Interim Report on Cooperation between JHA Agencies, doc. 5816/10, 29.01.2010.

18 Consejo de la Unión Europea, Summary of discussions of the meeting of the JHA Agencies (18 June 2008), doc. 11843/09, 09.07.2009.

19 Consejo Europeo, El Programa de Estocolmo: una Europa abierta y segura que sirva y proteja al ciudadano, (DO C-115/1, 04.05.2010), p. 6.

20 Consejo, doc. 5816/10, 29.01.2010.

21 Ibid.

22 Consejo de la Unión Europea, Final Report on the cooperation between JHA Agencies, doc. 8387/10, 09.04.2010, pp. 2-3. 
formación de expertos, el aumento de la sensibilización acerca de las tareas de las agencias, así como la creación de un enfoque común de gobernanza, asuntos institucionales y relaciones internacionales ${ }^{23}$. Como resultado de las recomendaciones de estos dos informes, las agencias del ELSJ decidieron evaluar con regularidad el grado de implementación de las medidas bilaterales y multilaterales acordadas ${ }^{24} \mathrm{y}$ organizar reuniones para garantizar una coherencia y continuidad en sus tareas operativas ${ }^{25}$.

El Consejo Europeo, en su agenda para el periodo 2015-2020, volvió a insistir en la relevancia de promover la cooperación entre las instituciones y organismos de la UE que operan en el ELSJ ya que «el éxito o el fracaso en uno de esos campos depende de la actuación en los demás campos, así como de las sinergias con los ámbitos de actuación afines» ${ }^{26}$. El Consejo Europeo destacó que el papel de las agencias debe, de ahora en adelante, centrarse en transponer, implementar y armonizar los numerosos instrumentos legales y medidas adoptadas en el ELSJ ${ }^{27}$. Con este fin, Frontex, Easo y Europol comparten y actualizan sus prioridades a corto y largo plazo, su grado de consecución y ejecución, celebran reuniones conjuntas y forman parte de una red de agencias del ELSJ cuya presidencia es rotatoria. A este respecto, los directores ejecutivos de las agencias del ELSJ se reúnen anualmente junto con representantes de la Comisión Europea, la Comisión LIBE del Parlamento Europeo, la Presidencia del Consejo de la UE, la Secretaría General del Consejo, la Oficina Europea de Lucha contra el Fraude y el Servicio Europeo de Acción Exterior $^{28}$. Durante esta reunión se evalúa el logro de los objetivos comunes establecidos por las agencias para ese año, se presentan las prioridades para el año siguiente y se adopta un informe final que valora la cooperación multilateral de las agencias del ELSJ ${ }^{29}$.

23 Id.

24 Consejo de la Unión Europea, Report on the Cooperation between JHA Agencies in 2010, doc. 5675/11, 25.01.2011.

25 Ibid., p. 6.

26 Consejo Europeo, Conclusiones de la Presidencia (26-27 junio 2014), EUCO 79/14, 27.06.2014, p. 1. Véase, Consejo de la Unión Europea, The JHA Agencies Contribution on the new multiannual Justice and Home Affairs programme, doc. 7313/14, 10.03.2014.

27 Ibid., p. 3.

28 Véanse Consejo de la Unión Europea, Heads of JHA Agencies meeting, 3-4 November 2015, doc. 14779/15, 01.12.2015 y Consejo de la Unión Europea, Heads of JHA Agencies meeting, 14 November 2016, doc. 15580/16, 14.12.2016.

29 Véanse Final Report of the JHA Agencies Network in 2015, Joint conclusions of the Heads of JHA Agencies meeting on 3-4 November 2015, Tallin, noviembre de 2015; Consejo 
En definitiva, mientras que la cooperación multilateral de las agencias del ELSJ ha sido especialmente incentivada desde la entrada en vigor del Tratado de Lisboa y la adopción del Programa de Estocolmo, sus interacciones han consistido en una coordinación informal, una cooperación a nivel estratégico y un intercambio de información y análisis de riesgos. Solo en los últimos años, Frontex, Easo y Europol han comenzado a cooperar operativamente sobre el terreno con el fin de asistir de manera coordinada y coherente a aquellos Estados miembros que experimentan una presión migratoria extraordinaria y repentina en sus sistemas de registro, acogida y asilo. El enfoque de los hotspots introducido en 2015, objeto de estudio en las dos siguientes secciones, es el mejor exponente de la reciente cooperación multilateral operativa y reforzada de Frontex, Easo y Europol, pues, por primera vez, un marco operativo común requiere formalmente a estas agencias que cooperen sobre el terreno.

\section{LA APROXIMACIÓN DE LOS HOTSPOTS: UNA COOPERACIÓN REFORZADA, MULTILATERAL Y OPERATIVA ENTRE FRONTEX, EASO Y EUROPOL}

Ante el exponencial aumento de migrantes irregulares que arribaron a las costas europeas durante el año 2015, la Comisión Europea adoptó el 13 de mayo la Agenda Europea de Migración, cuyo objetivo se centró en diseñar una estrategia común para coordinar la acción de los Estados miembros, las instituciones de la UE, las agencias del ELSJ, las organizaciones internacionales, la sociedad civil, las autoridades locales y terceros países ${ }^{30}$. Entre todos estos actores, la Comisión puso especial énfasis en reforzar el papel de Frontex, Easo y Europol en la efectiva implementación de las medidas previstas en la agenda $^{31}$.

En los hotspots, Frontex, Easo y Europol han de registrar, procesar e identificar a los migrantes que llegan a las costas de los Estados miembros, proporcionar a las autoridades nacionales infraestructuras y recursos humanos, asistir

de la Unión Europea, EU Justice and Home Affairs Agencies' cooperation in 2016 - Final report, doc. 15579/16, 16.12.2016.

30 Comunicación de la Comisión, Una Agenda Europea de Migración, COM(2015) 240 final, 13.05.2015.

31 El concepto de los hotspots fue originariamente diseñado en un estudio solicitado por la Comisión Europea en 2014 acerca de la viabilidad de establecer un Sistema Europeo de Guardias de Fronteras. Véase Unisys, Study on the feasibility of the creation of a European System of Border Guards to control the external borders of the Union, 16.06.2014, pp. 24-27. Disponible en: https://bit.ly/2eUQMWV. 
en las investigaciones destinadas a desmantelar las redes de trata y tráfico ilegal de migrantes y canalizar a los migrantes al procedimiento (de asilo, retorno o reubicación) que según su situación personal corresponda ${ }^{32}$. Dada la reciente creación, la falta de transparencia, la aún muy limitada atención doctrinal y la dispersión de la información relativa a los hotspots, esta sección se centra en explorar sus características, objetivos y funcionamiento. Especial atención se presta en este estudio a las concretas tareas operativas que les han sido conferidas a Frontex, Easo y Europol en los hotspots y las actividades que desarrollan en la práctica sobre el terreno.

\section{DEFINIENDO EL ENFOQUE DE LOS HOTSPOTS}

Los hotspots son áreas geográficas sujetas a flujos migratorios mixtos repentinos, específicos y excepcionales que los sistemas nacionales de fronteras y asilo no pueden procesar de manera efectiva ${ }^{33}$. El Estado miembro interesado, tras haber evaluado la situación en sus fronteras exteriores y haber tomado en consideración los análisis de riesgos preparados por Frontex y Easo, puede solicitar el establecimiento de uno o varios hotspots en su territorio ${ }^{34}$.

Los procedimientos de trabajo normalizados (PTN), adoptados por el Ministerio del Interior italiano en 2016 y que orientan (no son vinculantes) el funcionamiento de los hotspots italianos, proporcionaron una definición estructural y organizativa de los mismos. Los hotspots son estructuralmente áreas geográficas donde los migrantes son desembarcados, registrados, identificados y canalizados a los procedimientos de asilo, reubicación o retorno ${ }^{35}$. Desde un punto de vista organizativo, los hotspots constituyen un método de trabajo en equipo en el que las autoridades nacionales cooperan estrechamente con las agencias europeas con el fin de garantizar una gestión efectiva y plenamente operativa de los flujos migratorios mixtos a los que se ven sujetas ${ }^{36}$. La misión de los hotspots consiste, por tanto, en proporcionar una plataforma para que Frontex, Easo y Europol

32 El enfoque de los hotspots se adapta a la situación migratoria a la que está sujeto cada Estado miembro y a las particularidades de su sistema nacional de asilo y control fronterizo. A este respecto véanse Asylum Information Database, Country Report: Greece, 2016, disponible en: https://bit.ly/2nwd9nA y Asylum Information Database, Country Report: Italy, 2016, disponible en: https://bit.ly/2832r83.

33 Explanatory note on the "Hotspot" approach, 15.07.2015, p. 3. Disponible en: https:// bit.ly/1N46jcT. .

34 Standard Operating Procedures Applicable to Italian Hotspots, 2016, p. 4. Disponible en: http://bit.ly/2kt9JBX.

35 Ibid., p. 4.

36 Ibid., p. 4. 
coordinen ágilmente sus tareas operativas cuando asisten a aquellos Estados miembros que no pueden gestionar suficientemente por sí mismos la incesante llegada de flujos migratorios mixtos. Además, la aproximación de los hotspots aumenta el impacto sobre el terreno de la asistencia operativa que prestan Frontex, Easo y Europol, se evitan duplicaciones entre estas a la hora de asistir a los Estados miembros y se refuerza su visibilidad ${ }^{37}$.

La Agenda Europea de Migración adoptada por la Comisión Europea en 2015 que introdujo la aproximación de los hotspots indicó que en estos

la Oficina Europea de Apoyo al Asilo (OEAA), Frontex y Europol trabajarán sobre el terreno con los Estados miembros situados en primera línea para proceder rápidamente a la identificación, el registro y la toma de huellas dactilares de los migrantes. Los trabajos de las agencias se complementarán mutuamente. Los solicitantes de asilo serán inmediatamente canalizados a un procedimiento de asilo en el que los equipos de apoyo de la OEAA ayudarán a tramitar lo más rápidamente posible los casos de asilo. Para las personas que no necesitan protección, Frontex ayudará a los Estados miembros coordinando el retorno de los migrantes irregulares. Europol y Eurojust ayudarán al Estado miembro de acogida en sus investigaciones con vistas al desmantelamiento de las redes de tráfico y trata de seres humanos ${ }^{38}$.

Aparte de la breve descripción anterior de las funciones de las agencias del ELSJ en los hotspots y una nota explicativa enviada posteriormente por el comisario europeo Avramopoulos a los ministros de Justicia y Asuntos de Interior el 15 de julio de $2015^{39}$, no se ha propuesto aún un marco legal que regule específicamente el enfoque de los hotspots (Tribunal de Cuentas Europeo, 2017: 16; Neville et al., 2016: 26). Esto es, hasta la fecha únicamente el Reglamento 2016/1624, que refuerza las tareas conferidas originariamente a Frontex, describe de forma concisa y sectorial el funcionamiento de los hotspots y delimita someramente las tareas de las agencias en los mismos. Así, el art. 2 del Reglamento 2016/1624 define un hotspot como «una zona en la que el Estado miembro de acogida, la Comisión, los organismos de la Unión competentes y los Estados miembros participantes cooperan con el objeto de gestionar un reto migratorio desproporcionado, existente o potencial, caracterizado por un aumento significativo del número de migrantes que llegan a las fronteras exteriores». De acuerdo con el art. 18 del Reglamento 2016/1624, en estas zonas geográficas caracterizadas por flujos migratorios mixtos se deben desplegar equipos de apoyo a la gestión de la migración. Estos equipos estarán principalmente compuestos

37 Explanatory note on the "Hotspot" approach, pp. 5-6.

38 Una Agenda Europea de Migración, COM(2015) 240 final, 13.05.2015, p. 6.

39 Explanatory note on the "Hotspot" approach. 
por expertos de Frontex, Easo y Europol, quienes proporcionarán un apoyo operativo a los Estados miembros que lo soliciten ${ }^{40}$.

Si bien Easo viene participando en los hotspots desde su creación en 2015 mediante el despliegue de equipos de apoyo al asilo, es el Reglamento propuesto por la Comisión Europea el que describe someramente el papel de esta agencia en los hotspots, así como su participación en los equipos de apoyo a la gestión de la migración ${ }^{41}$. Las concretas tareas operativas que la sucesora de Easo, la AEA, desarrollará en los hotspots aún están siendo objeto de negociación y, muy particularmente, si la agencia podrá, a petición de los Estados miembros, examinar las solicitudes de protección internacional ${ }^{42}$. Por último, a diferencia del Reglamento 2016/1624 de Frontex y el futuro marco normativo de Easo, el nuevo Reglamento 2016/794 de Europol no menciona las funciones de esta agencia en los hotspots, ni el creciente apoyo operativo que presta a los Estados miembros en sus investigaciones relativas al tráfico ilegal de migrantes y trata de seres humanos (Blasi Casagran, 2018: 333-357).

Tampoco existe referencia oficial alguna a la base jurídica para el establecimiento del enfoque de los hotspots. A este respecto, la doctrina ha concluido que los arts. 78.3 y 80 del Tratado de Funcionamiento de la Unión Europea (TFUE) constituyen el fundamento para el establecimiento de hotspots en el territorio de un Estado miembro (Moreno-Lax, 2015: 12; Neville et al., 2016: $26)^{43}$. Los arts. 78.3 y 80 TFUE indican que en aquellas situaciones en las que un Estado miembro afronte una afluencia repentina y excepcional de migrantes irregulares en sus fronteras exteriores, la UE podrá adoptar medidas provisionales en beneficio del Estado miembro afectado de conformidad con los principios de solidaridad y reparto equitativo de la responsabilidad.

40 Véase Comunicación de la Comisión, Gestión de la crisis de los refugiados: medidas operativas, presupuestarias y jurídicas inmediatas en el marco de la Agenda Europea de Migración, anexo 2, COM(2015) 490 final, 23.09.2015.

41 Propuesta de Reglamento del Parlamento Europeo y del Consejo relativo a la Agencia de Asilo de la Unión Europea y por el que se deroga el Reglamento (UE) 439/2010, COM(2016) 271 final, 4.5.2016.

42 Véase Consejo, Proposal for a Regulation of the European Parliament and of the Council on the European Union Agency for Asylum and repealing Regulation (EU) No 439/2010 - State of play and guidance for further work, doc. 10555/17, 27.06.2017.

43 «En paralelo con la labor mencionada de refuerzo de la protección de las fronteras exteriores de la UE, la creación de los puntos críticos en Grecia e Italia es un logro operativo tangible y un ejemplo concreto de la aplicación de los principios de solidaridad y responsabilidad al responder a la presión a la que se enfrentan estos Estados miembros», Comunicación de la Comisión relativa al cumplimiento de la Agenda Europea de Migración, $\operatorname{COM(2017)~} 558$ final, 27.09.2017, p. 13. 
El 27 de septiembre de 2017, la Comisión Europea, en su comunicación relativa al cumplimiento de la Agenda Europea de Migración, declaró, refiriéndose a los hotspots, que deben establecerse claramente sus funciones y responsabilidades, así como «una coordinación más efectiva sobre el terreno, en estrecha cooperación con los Estados miembros de acogida y en especial a través de los grupos de trabajo regionales de la UE, a los que debe darse un mandato claro» ${ }^{44}$. Sin embargo, la Comisión no ha propuesto la adopción de un marco legal que regule, tal y como ella misma parece sugerir, las funciones y responsabilidades de los actores implicados en los hotspots. Por el contrario, la Comisión sigue decantándose por medidas flexibles y no vinculantes que se limitan a orientar acerca de las funciones de Frontex, Easo y Europol en los hotspots ${ }^{45}$.

Conforme al art. 18 del Reglamento 2016/1624, la Comisión Europea estima que, una vez activada la aproximación de los hotspots, los PTN son el instrumento que ha de guiar el funcionamiento y delimitar las responsabilidades de las autoridades que participan en los mismos. En concreto, la Comisión recomienda que todo Estado miembro que solicite la activación de la aproximación de los hotspots adopte los PTN (en coordinación con la Comisión, las agencias europeas y las organizaciones internacionales implicadas en los mismos) siempre con anterioridad al inicio de las operaciones y, según corresponda en cada Estado miembro, mediante su desarrollo en un acto jurídico, una decisión gubernamental o ministerial ${ }^{46}$. Pese a la importancia que la Comisión confiere a los PTN, estos solo fueron adoptados por el Ministerio del Interior italiano una vez que los hotspots ya habían empezado a funcionar. Por otro lado, y hasta la fecha, Grecia carece de cualquier guía operativa que oriente acerca del funcionamiento de los hotspots establecidos en su territorio.

A nuestro parecer, los PTN no excluirían sino que deberían complementar un marco jurídico a nivel europeo que aclare las tareas operativas de Frontex, Easo y Europol, y el grado de cooperación y asistencia que prestan a aquellas autoridades nacionales sujetas a presiones migratorias excepcionales y repentinas en sus fronteras exteriores. Un instrumento jurídico debería regular la aproximación de los hotspots en su conjunto para así aumentar la transparencia, la seguridad jurídica y contribuir a clarificar las responsabilidades de cada uno de los actores implicados que se ven difuminadas como consecuencia de la cooperación reforzada que acontece en los hotspots. Los PTN,

\footnotetext{
$44 \quad$ Ibid., p. 12.

45 Commission Staff Working Document Best practices on the implementation of the hotspot approach, SWD(2017) 372 final, 15.11.2017.

46

Ibid.
} 
por su parte, son una herramienta adecuada y lo suficientemente flexible para pormenorizar el funcionamiento de cada uno de los hotspots y adaptarse a la volátil y diferente realidad migratoria existente en cada uno de ellos.

Además, la regulación de los hotspots a nivel europeo ayudaría a equilibrar las fuerzas entre las agencias participantes, ya que Frontex está desempeñando en los mismos un papel preponderante, lo que a su vez revela el carácter más favorable a la «securitización» de las fronteras exteriores que a la protección internacional de los migrantes que se imprime a los hotspots (Rijpma, 2016: 26; Santos Vara, 2018: 161). Como apunta Tsourdi (2017b: 5), la Administración europea reforzada en materia de migración, asilo y gestión fronteriza que pone de manifiesto la aproximación hotspot no es en sí misma problemática siempre que venga acompañada de suficientes garantías administrativas. En este sentido, entendemos que el articulado del instrumento legislativo que se encargase de regular los hotspots también debería incluir medidas destinadas a proteger y promover la protección de los derechos fundamentales de los migrantes y los solicitantes de asilo.

\section{FUNCIONAMIENTO Y DIVISIÓN DE TAREAS ENTRE FRONTEX, EASO Y EUROPOL EN LOS HOTSPOTS}

Los Estados miembros son los responsables de solicitar a la Comisión Europea o a las agencias del ELSJ el establecimiento de uno o varios hotspots en su territorio. De acuerdo con la nota explicativa sobre el enfoque de los hotspots, las agencias evaluarán lo más rápidamente posible la solicitud de todo Estado miembro que reciban y, bajo la coordinación de la Comisión, diseñarán un paquete de ayuda integral para las autoridades nacionales ${ }^{47}$. Posteriormente, el Estado miembro solicitante será responsable no solo de informar a la Comisión sobre las áreas geográficas donde requiere los hotspots y los recursos nacionales que se les asignarán, sino también de adoptar los PTN que gobernarán los hotspots y de designar un organismo encargado de supervisar y coordinar todas las operaciones en los mismos ${ }^{48}$. Esto es, los hotspots se coordinan de manera centralizada en el propio territorio del Estado miembro solicitante mediante un centro de coordinación interinstitucional que aúna a las autoridades nacionales y locales competentes, la Comisión, las agencias del ELSJ y las principales organizaciones internacionales que trabajan sobre el terreno (Tribunal de Cuentas Europeo, 2017: 34).

47 Explanatory note on the "Hotspot" approach, p. 3.

48 Commission Staff Working Document Best practices on the implementation of the hotspot approach, SWD(2017) 372 final, 15.11.2017, pp. 1-2. 
Tan pronto como los hotspots entran en funcionamiento, se crea un Grupo Operativo Regional de la Unión Europea (EURTF), presidido por la Comisión Europea, encargado de promover el intercambio de información y coordinar sobre el terreno las tareas operativas de las autoridades nacionales y de los equipos de apoyo a la gestión de la migración desplegados por las agencias (Tribunal de Cuentas Europeo, 2017: 35$)^{49}$. La participación de Frontex, Easo y Europol en el EURTF facilita la aplicación del enfoque de los hotspots, ya que refuerza la presencia operativa de las agencias sobre el terreno, promueve su cooperación con las autoridades nacionales competentes, mejora el intercambio rápido de información entre las partes interesadas y promueve la aplicación efectiva y uniforme del acervo de la UE en materia de migración, asilo y gestión de fronteras a nivel nacional y local ${ }^{50}$.

\section{EASO}

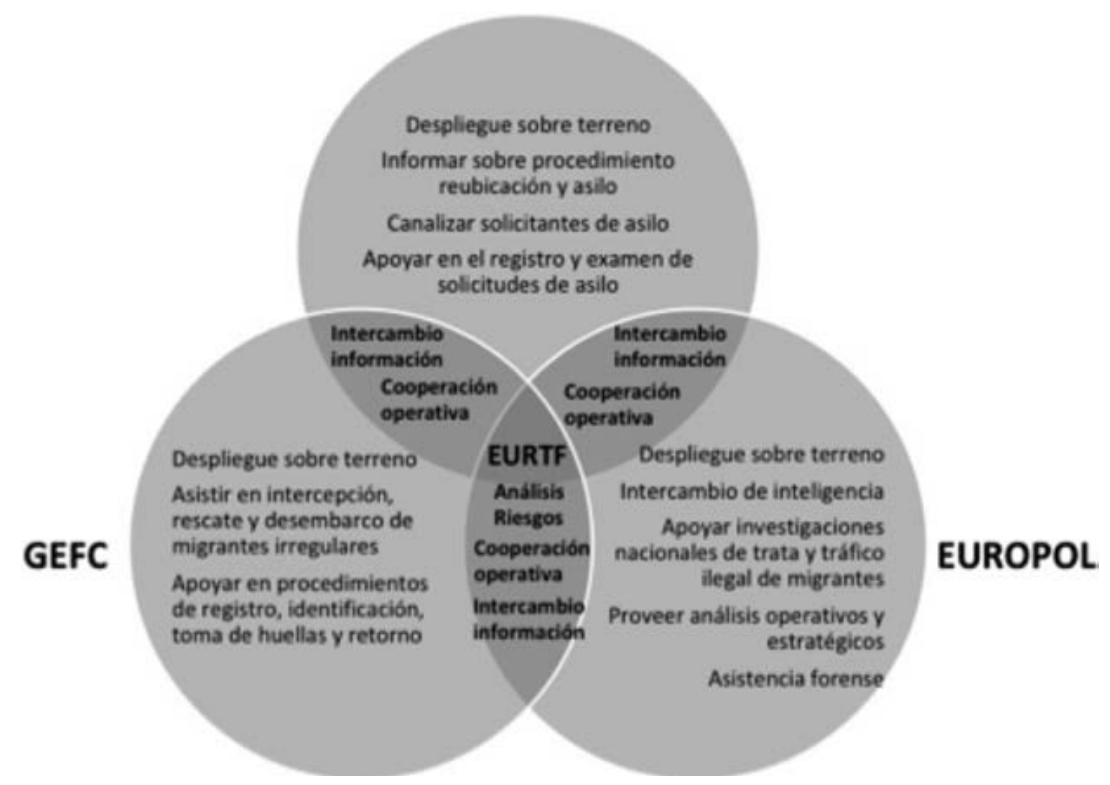

Figura 1: Funcionamiento de los hotspots.

Fuente: elaboración propia.

49 Véanse Comunicación de la Comisión sobre el estado de ejecución de las medidas prioritarias en el marco de la Agenda Europea de Migración, COM(2016) 85 Final, 10.2.2016 y Easo, Hotspot-Relocation Operating Plan to Italy, Easo/COS/2015/945/ IT/2015, 2015.

50 Véanse Frontex, General Annex of the Operational Plan - Joint Maritime Operations, 2016, pp. 18-19 (en el archivo del autor) y Antonakaki, et al. (2016). 


\subsection{La asistencia operativa de Frontex en el registro, la identificación y el retorno de los migrantes irregulares}

Frontex desempeña una triple función operativa en los hotspots: registro e investigación (toma de huellas dactilares y determinación de la identidad y nacionalidad de los migrantes recién llegados), interrogación e información (asistencia a las investigaciones nacionales de tráfico ilegal de migrantes y trata de seres humanos) y retorno. Estas tareas vienen expresamente reseñadas en el art. 18.4 del Reglamento 2016/1624 de Frontex que regula el despliegue de los equipos de apoyo a la gestión de la migración en los hotspots.

Respecto a las tareas de registro e investigación debe señalarse que la asistencia de Frontex comienza antes de que los migrantes irregulares hayan llegado a las costas de los Estados miembros. Mediante patrulleras, helicópteros y aeronaves ${ }^{51}$, Frontex apoya activamente a las autoridades nacionales competentes en sus operaciones de búsqueda, rescate, vigilancia, detección temprana y prevención de cruces fronterizos irregulares ${ }^{52}$. Además, y de acuerdo con los PTN aplicables a los hotspots italianos, el jefe del equipo de Frontex desplegado en un concreto hotspot contactará con el funcionario de enlace de la agencia que esté a bordo del buque o con el capitán encargado de la operación marítima para recibir con antelación todos aquellos datos que permitan a la agencia preparar las actividades de registro que tendrán lugar tan pronto como los migrantes irregulares sean desembarcados ${ }^{53}$.

Frontex debe proporcionar a los migrantes desembarcados información relativa a los procedimientos de asilo y reubicación, así como asistir a las autoridades nacionales en su registro e identificación. Un aspecto clave durante esta fase consiste en determinar la nacionalidad de los migrantes, pues la mayoría viaja sin documentos o con falsificaciones. Las autoridades nacionales junto con los funcionarios desplegados por Frontex analizan, con la ayuda de un intérprete y un experto en fraude documental puestos a disposición por la agencia, el país de origen de los migrantes.

La determinación de la nacionalidad de los migrantes irregulares se lleva a cabo mediante el examen de los documentos que traían consigo, y en su defecto, mediante una entrevista (Guild et al., 2017: 55). Frontex emite una

51 Hasta la fecha puestos a su disposición por los Estados miembros aunque el nuevo Reglamento 2016/1624 prevé que estos puedan también ser adquiridos o arrendados por la propia agencia.

52 Frontex, Evaluation Report 2016 - JO EPN Triton 2016, 2016, p. 10 (en el archivo del autor). Véase Franco García (2018: 13-54).

53 Standard Operating Procedures Applicable to Italian Hotspots, 2016, p. 23. 
recomendación no vinculante para el Estado miembro en la que se detalla la evaluación realizada y la nacionalidad que se le presume al migrante irregular. Pese a que las autoridades nacionales son exclusivamente competentes a la hora de adoptar una decisión final respecto al país de origen del migrante irregular, estas tienden a basar sus decisiones en la evaluación y recomendación elaborada por Frontex. Ello es debido a la extraordinaria presión migratoria a la que están sujetas en los hotspots, así como a la falta de recursos nacionales suficientes para identificar y determinar la nacionalidad de los numerosos migrantes irregulares que arriban diariamente (European Council for Refugees and Exiles, 2016a: 40).

Tan pronto como la nacionalidad de los migrantes es dirimida, los equipos de apoyo de Frontex desplegados se encargan de rellenar un documento de registro donde se detalla la nacionalidad, la edad y la lengua del migrante, así como su intención o no de solicitar asilo ${ }^{54}$. Posteriormente, los oficiales de toma de huellas dactilares de Frontex deben asistir a las autoridades nacionales en la toma de las huellas de los migrantes desembarcados y su registro en el sistema Eurodac. Los migrantes han de ser informados acerca del uso que se dará al registro de sus huellas dactilares y su obligación de introducirlas en Eurodac. En caso de que los migrantes se opongan a que sus huellas sean registradas, únicamente los funcionarios nacionales podrán aplicar el nivel mínimo de fuerza para obtenerlas ${ }^{55}$.

Otra función principal que Frontex desarrolla en los hotspots consiste en recabar información sobre las rutas migratorias, trata de seres humanos y tráfico ilegal de migrantes. De acuerdo con los PTN de los hotspots italianos, los expertos de Frontex deben recabar cualquier información relevante que se desprenda de los efectos personales y otros objetos que los migrantes irregulares lleven consigo al ser interceptados. Frontex, en estrecha cooperación con Europol y las autoridades nacionales, analizará los objetos incautados con el objetivo de establecer cualquier vínculo con redes especializadas en migración ilegal y trata. En concreto, Frontex puede entrevistar a aquellos migrantes irregulares que voluntariamente se presten a detallar las rutas empleadas para llegar a Europa, las razones para emprender su viaje y el modus operandi de las redes de trata y tráfico ilegal de migrantes. Frontex debe recopilar y compartir con Europol toda la información recabada durante las entrevistas ${ }^{56}$.

\footnotetext{
54 Ibid., p. 40.

55 Frontex, General Annex of the Operational Plan-Joint Maritime Operations, 2016, p. 28 (en el archivo del autor).

56 Ibid, p. 20.
} 
Es importante apuntar que, a diferencia de la obligación que tienen los migrantes irregulares de participar en las entrevistas que se llevan a cabo durante la fase de registro para determinar su país de origen, las entrevistas destinadas a recabar información sobre las redes de trata y tráfico ilegal de migrantes son voluntarias y anónimas. A este respecto, el relator especial sobre los derechos humanos de los migrantes recomendó que estas entrevistas no deberían tener lugar a la llegada de los migrantes a los hotspots, ya que a menudo estos vienen traumatizados por el viaje y dichas entrevistas pueden aumentar su temor a las autoridades y conducirles a ocultar sus necesidades de protección o su situación de vulnerabilidad ${ }^{57}$.

Por último, tan pronto como los migrantes irregulares son identificados y registrados, Frontex se encarga de asistir a las autoridades nacionales respecto a aquellos migrantes que no pueden permanecer en la UE de manera regular y deben ser expulsados. Frontex asiste al Estado miembro durante el procedimiento de retorno tanto en la fase previa de organización de su retorno y coordinación con las autoridades del país de origen del migrante, como durante la fase de retorno propiamente dicha (arts. 27-33 del Reglamento 2016/1624).

Frontex despliega, por tanto, cinco categorías diferentes de expertos en los hotspots: expertos en registro, oficiales para la toma de huellas dactilares, expertos en el análisis de documentos de identidad, entrevistadores y oficiales de apoyo al retorno. Si bien todos estos expertos proporcionan en la práctica un apoyo destacado a las autoridades nacionales en la vigilancia y control de sus fronteras, el Estado miembro debe, en principio, coordinar las tareas que desarrollan los equipos desplegados por Frontex y supervisar todas sus operaciones.

\subsection{La asistencia de Easo en el registro y el examen de las solicitudes de asilo}

La misión principal de Easo en los hotspots consiste en proporcionar asistencia operativa a aquellas autoridades nacionales que reciban un alto número de solicitudes de asilo ${ }^{58}$. Easo se encarga de desplegar equipos de asilo con el fin de evitar la acumulación de procedimientos de protección internacional y de diseñar un sistema nacional de asilo sostenible y eficiente (Papageorgiou, 2017: 37). De acuerdo con los planes operativos elaborados por Easo, el papel

57 Human Rights Council, Report of the Special Rapporteur on the human rights of migrants on his mission to Greece, A/HRC/35/25/Add.2, 24.04.2017, apdo. 41.

58 Easo, Single Programming Document: Multiannual Programming 2017-2019 and Work Programme 2017, 2016, p. 10. 
de la agencia en los hotspots debe centrarse en tres aspectos: 1) proporcionar información detallada a los recién llegados sobre el procedimiento de reubicación y asilo; 2) canalizar a los solicitantes de asilo hacia el procedimiento de asilo, reubicación o protección internacional y 3) asistir a las autoridades nacionales con el registro y el examen de las solicitudes de asilo, la preparación de los expedientes y la tramitación de las solicitudes ${ }^{59}$.

Los equipos de asilo de Easo, en estrecha colaboración con el Alto Comisionado de las Naciones Unidas para los Refugiados (ACNUR), deben informar a los migrantes que lleguen a los hotspots sobre las fases y el funcionamiento del procedimiento de asilo y reubicación. Los nacionales de terceros países también son asesorados acerca de dónde y cómo pueden presentar sus solicitudes, así como la necesidad de cooperar con las autoridades nacionales para asegurar un efectivo examen de su solicitud ${ }^{60}$. En la práctica, Easo ha jugado un significativo papel en la implementación de las decisiones del Consejo sobre reubicación hasta la desaparición del programa a finales de 2017. Específicamente, la agencia apoyaba a los Estados miembros en la identificación de aquellos solicitantes elegibles para ser reubicados, el registro de sus solicitudes, el desarrollo de entrevistas y la preparación de las decisiones de reubicación ${ }^{61}$.

La tarea operativa más controvertida que Easo lleva a cabo en los hotspots griegos es el apoyo a las autoridades nacionales en la tramitación de las solicitudes de asilo. Dado el colapso del sistema de asilo griego, Easo ha asumido de facto la importante tarea de gestionar la entrevista de admisibilidad de las solicitudes de asilo. Conforme al plan operativo acordado por Easo y Grecia para 2018, la asistencia operativa de Easo en los hotspots comprende el registro y el análisis de la elegibilidad y admisibilidad de las solicitudes asilo presentadas, así como la identificación y evaluación de la vulnerabilidad de los solicitantes ${ }^{62}$.

Desde que se adoptó la Declaración entre la UE y Turquía ${ }^{63}$, Easo se fue gradualmente involucrando en el examen de las solicitudes de asilo mediante

59 Easo, Hotspot - Relocation Operating Plan to Italy, EASO/COS/2015/945/IT/2015, 12.12.2015 y Easo, Hotspot Operating Plan to Greece, EASO/COS/2015/677 EL/1226, 30.09.2015. Véase, Easo, Operating Plan Agreed by Easo and Italy, 15.12.2017 y Easo, Operating Plan Agreed by Easo and Greece, 13.12.2017.

60 Easo, Hotspot Operating Plan to Greece - Amendment No 2, EASO/COS/2016/391, 01.04.2016.

61 Easo, Consolidated Annual Activity Report of EASO 2016, 13.06.2017, p. 20. Véase Guild et al. (2017: 53).

62 Easo, Operating Plan Agreed by Easo and Greece, 13.12.2017.

63 Consejo Europeo, Declaración UE-Turquía, comunicado de prensa: 144/16, 18.03.2016. En particular, los ministros de Asuntos Exteriores determinaron que los 
la realización de las entrevistas de admisibilidad, la redacción de opiniones y la adopción de recomendaciones a las autoridades helenas al respecto ${ }^{64}$. El procesamiento conjunto de las solicitudes de asilo está expresamente previsto en el art. 60.4, apdo. b, de la Ley griega n. 4375 de 3 de abril de 2016, que establece que si bien la policía helena o las fuerzas armadas son responsables en los hotspots del registro de las solicitudes de asilo, Easo puede asistir a las autoridades nacionales mediante la realización de entrevistas con los solicitantes de asilo ${ }^{65}$.

El art. 60.4 de la Ley griega n. ${ }^{\circ} 4375$ de 3 de abril de 2016 fue reformado por la Ley griega n. 4399 de 22 de junio de $2016^{66}$, la cual amplió aún más las funciones conferidas a Easo, pues en los hotspots griegos la agencia fue facultada para conducir de manera autónoma las entrevistas a los solicitantes de asilo (Tsourdi, 2016: 1023; Tsourdi, 2017a: 1-20). Es decir, los funcionarios de Easo desplegados en los hotspots griegos desempeñan tareas clave como son entrevistar a los solicitantes de asilo, evaluar su caso y elevar una recomendación a las autoridades griegas acerca de la admisibilidad de la solicitud de asilo (Papageorgiou, 2017: 38; European Council for Refugees and Exiles, 2016b: 38). Aunque esta recomendación de Easo no es vinculante para los funcionarios griegos, la opinión de Easo presenta efectos cuasi vinculantes en la práctica, pues el Servicio de Asilo griego, dada la presión migratoria a la que está sujeto, se limita a ratificar la recomendación emitida por la agencia y no entra a evaluar el fondo de la solicitud.

retornos a Turquía desde Grecia debían intensificarse y que la asistencia de la UE a través de Frontex y Easo debía priorizarse con el objetivo de garantizar tales retornos; Consejo de la Unión Europea, Outcome of the Council Meeting 3473rd Council meeting Justice and Home Affairs Luxembourg, 9 and 10 June 2016, doc. 9979/16, p. 10. Véase Gkliati (2017: 81-123).

64 Easo, Hotspot Operating Plan to Greece - Amendment No 2, EASO/COS/2016/391, 01.04.2016, p. 3; Easo, Special Operating Plan to Greece, EASO/DOP/OU/2016/1812, 15.12.2016, p. 9 y Easo, Operating Plan Agreed by Easo and Greece, 13.12.2017, p. 13. Véase Papageorgiou (2017: 38).

65 Greece Law No. 4375 of 2016 on the organization and operation of the Asylum Service, the Appeals Authority, the Reception and Identification Service, the establishment of the General Secretariat for Reception, the transposition into Greek legislation of the provisions of Directive 2013/32/EC, 03.04.2016. Disponible en: http://www.refworld.org/docid/573ad4cb4.html. La referencia a esta ley fue encontrada en Papageorgiou (2017: 7).

66 Law 4399, Institutional framework for establishing Private Investment Aid schemes for the country's regional and economic development - Establishing the Development Council and other provisions, 22.06.2016, p. 6905. Disponible en: https://bit.ly/2OrHZwQ. La referencia a esta ley fue encontrada en Papageorgiou (2017: 7). 


\subsection{La asistencia de Europol en la prevención y lucha contra el tráfico ilegal de migrantes y la trata de seres humanos}

Europol también está presente en los hotspots y participa activamente con Frontex y Easo en el EURTF. La misión de Europol en los hotspots es triple: facilita el intercambio de información, verifica y coteja dicha información en sus bases de datos, y despliega equipos de expertos sobre el terreno con el fin de asistir a los Estados miembros en sus investigaciones nacionales de tráfico ilegal de migrantes y la trata de seres humanos. Europol es responsable de fomentar un rápido intercambio de información, de apoyar a las investigaciones nacionales, de conducir análisis operativos y estratégicos y de proporcionar apoyo forense ${ }^{67}$.

Sin embargo, a diferencia de los reglamentos de Frontex y de la propuesta de reglamento de la futura AEA que expresamente regulan el apoyo de estas agencias a los Estados miembros en los hotspots, el nuevo Reglamento 2016/794 de Europol no hace referencia a la función operativa que la agencia desempeña en estos. Dado que tampoco se ha adoptado un marco legal específico para los hotspots, existe un substancial grado de inseguridad jurídica en torno a las concretas tareas operativas que Europol puede desarrollar sobre el terreno.

La única referencia que el nuevo Reglamento 2016/794 de Europol hace a las tareas operativas de la agencia es la posibilidad de establecer centros especializados para coordinar, organizar y ejecutar acciones de investigación que ayuden a los Estados miembros en la lucha contra la delincuencia transnacional y el terrorismo. En particular, el art. 4.1, apdo. 1, del Reglamento 2016/794 establece que Europol podrá «crear centros de asesoramiento especializados de la Unión para combatir determinados tipos de delitos». El Consejo de Administración de Europol, a propuesta del director ejecutivo, es el encargado de decidir acerca de la creación de estos centros (art. 11.1, apdo. s, del Reglamento 2016/794). Hasta la fecha, Europol ha establecido tres centros: el Centro Europeo de Ciberdelincuencia (EC3), el Centro Europeo Contra el Tráfico Ilícito de Migrantes (EMSC) y el Centro Europeo de Lucha contra el Terrorismo (ECTC).

La creación del EMSC, al igual que los hotspots, tuvo su origen en la Agenda Europea de la Migración. Uno de los objetivos de la agenda es la lucha contra las redes de trata y tráfico ilegal de migrantes ya que la mayoría de los migrantes irregulares que llegan a Europa recurren a servicios de facilitación proporcionados

67 Europol, General Report on Europol Activities 2015, 2016, p. 11. Véase Standard Operating Procedures Applicable to Italian Hotspots, 2016, pp. 21-22. 
por grupos delictivos ${ }^{68}$. Precisamente, en marzo de 2015, Europol puso en marcha un Equipo Operativo Conjunto (JOT) para luchar contra la migración irregular y hacer frente a las mafias que operan en el Mediterráneo (MARE). A este respecto, la Comisión Europea indicó que «Europol procederá inmediatamente a reforzar su operación conjunta de información marítima (JOT MARE), de reciente creación, y su punto de coordinación sobre el tráfico de migrantes» ${ }^{69}$. El JOT MARE tuvo como cometido emprender acciones coordinadas contra los facilitadores basadas en el intercambio de inteligencia, apoyar a los Estados miembros en el inicio de nuevas investigaciones y garantizar un intercambio intensivo de información con Frontex, Interpol y los expertos nacionales adscritos al JOT ${ }^{70}$.

Tras la presentación de la Agenda Europea de Migración, la Comisión Europea publicó el Plan de Acción de la UE contra el Tráfico Ilícito de Migrantes (2015-2020) en el que se propuso que, «en el marco de JOT MARE, debería desplegarse un equipo operativo ad hoc para mejorar la recopilación de información y las capacidades operativas ${ }^{71}$. Paralelamente, el Parlamento Europeo y el Consejo también subrayaron que los Estados miembros han de colaborar estrechamente con Europol para abordar y desarticular las redes de trata y tráfico ilegal de personas ${ }^{72}$. Finalmente, el 22 de febrero de 2016, Europol inauguró el EMSC, en el que se integró el JOT Mare ${ }^{73}$.

El EMSC de Europol brinda a las autoridades nacionales tanto apoyo físico sobre el terreno como virtual para combatir el contenido online destinado

68 Europol, Migrant Smuggling in the EU, 2016. Disponible en: https://bit.ly/2EgCEUc.

69

70 iterranean, 17.03.2015. Disponible en: http://bit.ly/2zdUi7f. Véase Europol, Hit on Migrant Smuggling and Human Trafficking Ring Operating via the Mediterranean, 03.11.2015. Disponible en: http://bit.ly/2A9oL3e.

71 Comunicación de la Comisión, Plan de Acción de la UE contra el tráfico ilícito de migrantes (2015-2020), COM(2015) 285 final, 27.05.2015, p. 5.

72 Véanse: Resolución del Parlamento Europeo, de 29 de abril de 2015, sobre las recientes tragedias en el Mediterráneo y las políticas de inmigración y asilo de la UE [2015/2660(RSP)]; Resolución del Parlamento Europeo, de 10 de septiembre de 2015, sobre la migración y los refugiados en Europa [2015/2833(RSP)]; Resolución del Parlamento Europeo, de 12 de abril de 2016, sobre la situación en el mar Mediterráneo y necesidad de un enfoque integral de la Unión sobre la migración [2015/2095(INI)]; Consejo de la Unión Europea, Council conclusions on migrant smuggling, doc. 6995/16, 10.03.2016.

73 Europol, Europol Launches the European Migrant Smuggling Centre, 22.02.2016. Disponible en: http://bit.ly/2eL3W87. 
a facilitar el tráfico de migrantes ${ }^{74}$. La asistencia del EMSC se divide en cinco áreas principales de acción: 1) apoyo operacional, coordinación y transferencia de buenas prácticas; 2) apoyo estratégico a los Estados miembros; 3) intercambio de información entre los Estados miembros; 4) apoyo a EURTF y los hotspots; 5) despliegues sobre el terreno de los Equipos de Investigación Móvil (EMIST) y los Equipos de Análisis Móvil (EMAST). En concreto, los EMIST y EMAST de Europol son responsables de mejorar el análisis operativo y las investigaciones de los Estados miembros proporcionando una imagen completa, un intercambio sistemático y en tiempo real de información y buenas prácticas. Desde marzo de 2016, estos equipos han sido desplegados en diferentes hotspots para realizar controles secundarios de seguridad y apoyar a las autoridades griegas en sus investigaciones nacionales ${ }^{75}$.

Según el informe general sobre las actividades llevadas a cabo por Europol durante el año 2015, las tareas de la agencia en los hotspots consistieron en recopilar información en tiempo real de todos los procedimientos de desembarco y entrevistas, cotejar dicha información con las bases de datos de la agencia, proporcionar apoyo forense mediante el examen de dispositivos electrónicos y el escaneo de documentos, y apoyar a los investigadores nacionales y locales mediante la recopilación y el análisis de todo aquel material que pueda ser considerado relevante para la lucha contra el tráfico ilícito de migrantes, la trata y el terrorismo ${ }^{76}$.

\section{EL IMPACTO Y LA COMPATIBILIDAD DE LOS HOTSPOTS CON LOS REGLAMENTOS DE FRONTEX, EASO Y EUROPOL Y CON LA DOCTRINA DEL TJUE DE NO DELEGACIÓN}

\section{EL LIMITADO IMPACTO INICIAL DE LOS HOTSPOTS}

El impacto del enfoque se vio en sus inicios muy condicionado tanto por el retraso en la inauguración de los hotspots en Grecia e Italia como por el número insuficiente de expertos y recursos que los Estados miembros

74 Europol, European Migrant Smuggling Center - First Year Activity Report, 24.02.2017 y Europol, Two Years of EMSC. Activity Report. Jan 2017-Jan 2018, 20.04.2018.

75 Consejo de la Unión Europea, Conclusions of the Twelfth Annual Meeting of National Experts on Joint Investigation Teams (JITs) 15 and 16 June 2016, Eurojust, The Hague, doc. 12887/16, 05.10.2016, p. 3.

76 Europol, General Report on Europol Activities 2015, 2016, p. 11. Standard Operating Procedures Applicable to Italian Hotspots, 2016, p. 22. 
pusieron a disposición de las agencias. De los once hotspots cuya apertura estaba programada para diciembre de 2015, tan solo tres empezaron a funcionar a mediados de enero de 201677. Por un lado, de los cinco hotspots identificados por las autoridades griegas (Lesbos, Leros, Kos, Chios y Samos), solo el situado en la isla de Lesbos funcionaba de manera parcial siete meses después de la adopción de la Agenda Europea de Migración en mayo de 2015 $5^{78}$. Ante el retraso, la Comisión, a través de su Servicio de Apoyo a las Reformas Estructurales (un nuevo programa puesto en marcha desde el 1 de julio de 2015), ofreció a Grecia asistencia técnica especializada en la puesta en funcionamiento de los hotspots ${ }^{79}$. Por otro lado, la situación en Italia era algo mejor dado que de los cinco hotspots previstos (Lampedusa, Trapani, Pozzallo, Taranto y Messina), el de Lampedusa y Trapani abrieron un 22 de diciembre de $2015^{80}$. En 2017, cinco hotspots en Grecia y cuatro en Italia, con una capacidad de más de 7000 y 1500 plazas, respectivamente estaban operativos. Estos hotspots en total habían acogido a más de 261000 migrantes entre el 1 de enero de 2016 y el 15 de septiembre de $2017^{81}$.

La segunda principal limitación a la que se vieron sujetos los hotspots fue el insuficiente apoyo en forma de recursos humanos y equipos técnicos puestos a disposición de las agencias por parte de los Estados miembros. Como resultado de la adopción de la Agenda Europea de la Migración, Easo y Frontex solicitaron 370 y 776 oficiales adicionales respectivamente ${ }^{82}$. Europol, por su parte, anunció en 2016 su intención de desplegar en los hotspots un equipo de

77 Informe de la Comisión, Segundo informe sobre reubicación y reasentamiento, COM(2016) 222 final, 12.04.2016, p. 4; ACNUR (2016). Véase Neville (2016: 3340).

78 Comunicación de la Comisión, Informe de situación sobre el establecimiento de los puntos críticos en Grecia, COM(2015) 678 final, 15.12.2015, p. 5. Comunicación de la Comisión, Informe de situación sobre la ejecución del enfoque de hotspots en Grecia, COM(2016) 141 final, 04.03.2016.

79 Comunicación de la Comisión, Informe de situación sobre el establecimiento de los puntos críticos en Grecia, COM(2015) 678 final, 15.12.2015, p. 3.

80 Comunicación de la Comisión Informe sobre los progresos realizados en la implementación de los puntos críticos en Italia, $\operatorname{COM(2015)~} 679$ final, 15.12.2015.

81 Comunicación de la Comisión relativa al cumplimiento de la Agenda Europea de Migración, COM(2017) 558 final, 27.09.2017, p. 12.

82 Comunicación de la Comisión gestión de la crisis de los refugiados: estado de ejecución de las acciones prioritarias con arreglo a la Agenda Europea de Migración, $\operatorname{COM}(2015) 510$ final, 14.10 .2015 , p. 3. 
doscientos investigadores ${ }^{83}$. Sin embargo, estas cifras no fueron satisfechas con la necesaria premura de cara a cubrir las urgentes necesidades de las agencias en los hotspots ${ }^{84}$. Además, dado que el personal puesto a disposición de Frontex, Easo y Europol solo puede desplegarse durante cortos periodos de tiempo, las agencias están constantemente obligadas a solicitar expertos a los Estados miembros, lo que a su vez «implica una pérdida significativa de eficiencia, ya que los expertos recién llegados necesitan cierto tiempo para adaptarse y familiarizarse con la situación específica» (Tribunal de Cuentas Europeo, 2017: 32).

Aunque en 2017 y 2018 los compromisos de los Estados miembros con Frontex, Easo y Europol se mantuvieron por debajo de lo solicitado, las agencias vieron reforzados sus recursos significativamente ${ }^{85}$. Las operaciones conjuntas Poseidón y Tritón organizadas por Frontex proporcionaron respectivamente a Grecia e Italia apoyo en la aplicación efectiva del enfoque de los hotspots (Franco García, 2018: 42-45). Específicamente, en el marco de estas operaciones conjuntas Frontex desplegó 888 oficiales en Grecia y 407 en Ita$\operatorname{lia}^{86}$. La operación conjunta Tritón fue reemplazada y reforzada desde febrero

83 Europol, Europol Setting up Team of 200 Investigators to Deploy to Migration Hotspots, 12.05.2016. Disponible en: http://bit.ly/2nasaed. Finalmente, en 2016, el grupo inicial de 200 investigadores se redujo a un grupo de 116 oficiales invitados, 32 de los cuales se desplegaron en los hotspots griegos e italianos. A este respecto véanse: Quinto informe sobre los progresos realizados en la aplicación de la Declaración UE-Turquía, COM(2017) 204 final, 02.03.2017, p. 4 y Europol, European Migrant Smuggling Center First Year Activity Report, 2017, p. 15. Disponible en: https://bit.ly/2Ek7rQ9.

84 Comunicación de la Comisión, Informe de situación sobre el establecimiento de los puntos críticos en Grecia, $\operatorname{COM}(2015) 678$ final, 15.12.2015, p. 7; Informe de situación sobre la ejecución del enfoque de hotspots en Grecia, COM(2016) 141 final, 04.03.2016, p. 6; Comunicación de la Comisión, Informe sobre los progresos realizados en la implementación de los puntos críticos en Italia, COM(2015) 679 final, 15.12.2015, p. 3 .

85 Quinto informe sobre los progresos realizados en la aplicación de la Declaración UE-Turquía, $\operatorname{COM(2017)~} 204$ final, 02.03.2017, p. 4; Sexto informe sobre los progresos realizados en la aplicación de la Declaración UE-Turquía, $\operatorname{COM(2017)~} 323$ final, 13.06.2017, p. 3; Séptimo informe sobre los progresos realizados en la aplicación de la Declaración UE-Turquía, $\operatorname{COM(2017)~} 470$ final, 06.09.2017, p. 4; Comisión, Communication on Progress report on the Implementation of the European Agenda on Migration, $\operatorname{COM}(2018) 301$ final, 16.05.2018.

86 Quinto informe de la Comisión sobre la puesta en marcha de la Guardia Europea de Fronteras y Costas, $\operatorname{COM}(2017) 467$ final, 06.09.2017, p. 3. Véase el anexo de la Comunicación de la Comisión, Informe sobre los progresos realizados en la Agenda 
de 2018 con la nueva operación Themis, que aborda en mejor medida los patrones cambiantes de la migración en el Mediterráneo ${ }^{87}$. Easo, por su parte, desplegó, durante 2017, 232 expertos en los hotspots (107 trabajadores sociales, expertos en vulnerabilidad y en provisión de información sobre el procedimiento de asilo en la Unión, 42 empleados de Easo y 83 intérpretes) $^{88}$. A 30 de abril de 2018, Easo tenía desplegados 63 expertos nacionales, 27 funcionarios interinos y 85 intérpretes en Grecia y 38 expertos nacionales, 54 funcionarios interinos y 98 mediadores culturales en Italia ${ }^{89}$.

El despliegue de agentes invitados por parte de Europol en los hotspots también ha ayudado a las autoridades policiales nacionales a identificar perfiles de riesgo, luchar contra las redes de trata y tráfico y realizar controles de seguridad. Precisamente, en 2016 Europol recibió una subvención de asistencia de emergencia del Fondo de Seguridad Interior de 1,5 millones de euros para desplegar agentes invitados en los hotspots ${ }^{90}$. A finales de 2017, el grupo de agentes invitados en comisión de servicios, entrenados por Europol y desplegados en rotaciones trimestrales en los hotspots griegos e italianos, ascendió a 278 (Tribunal de Cuentas Europeo, 2017: 33) ${ }^{91}$. A 30 de abril de 2018, 13 agentes invitados de Europol y 2 miembros del personal de Europol estaban desplegados en los hotspots de Grecia y 15 agentes invitados y 3 de Europol en los italianos para realizar controles secundarios de seguridad ${ }^{92}$. Solo durante el mes de septiembre de 2016 los agentes invitados desplegados por Europol

Europea de Migración Guardia Europea de Fronteras y Costas, $\operatorname{COM}(2017) 669$ final, Annex 4, 15.11.2017.

87 Comision, Communication on Progress report on the Implementation of the European Agenda on Migration, $\operatorname{COM}(2018) 301$ final, 16.05.2018, p. 9.

88 Anexo de la Comunicación de la Comisión, Informe sobre los progresos realizados en la Agenda Europea de Migración Plan de Acción Conjunto sobre la aplicación de la Declaración UE-Turquía, COM(2017) 669 final, Annex 2, 15.11.2017, p. 1.

89 Comision, Communication on Progress report on the Implementation of the European Agenda on Migration, COM(2018) 301 final, 16.05.2018, pp. 6 y 9.

90 Parlamento Europeo, Draft Opinion of the Committee on Civil Liberties, Justice and Home Affairs for the Committee on Budgetary Control on discharge in respect of the implementation of the budget of the European Union Agency for Law Enforcement Cooperation (Europol) for the financial year 2016 [2017/2169(DEC)], 12.12.2017, p. 3.

91 Informe de la Comisión, Séptimo informe sobre los progresos realizados en la aplicación de la Declaración UE-Turquía, COM(2017) 470 final, 06.09.2017, p. 4.

92 Comision, Communication on Progress report on the Implementation of the European Agenda on Migration, COM(2018) 301 final, 16.05.2018, pp. 6 y 9. 
en los hotspots griegos realizaron controles secundarios de seguridad a 782 migrantes y durante el mes de octubre, a $1490^{93}$.

En definitiva, la lentitud en la puesta en marcha de los hotspots, la constante dependencia de las agencias de los recursos puestos a disposición por los Estados miembros, el deplorable estado de las instalaciones de acogida y la presión migratoria excepcional e incesante en las costas griegas e italianas hasta finales del año 2016 originó, en las propias palabras de la Comisión, que las instalaciones de los hotspots «no solo están masificadas, sino que presentan también condiciones materiales deficientes en términos de salubridad e higiene y acceso a servicios básicos tales como la asistencia sanitaria, en particular para los grupos vulnerables. La seguridad es insuficiente, y persisten las tensiones entre las diferentes nacionalidades» ${ }^{94}$.

A este respecto, los hotspots han sido negativamente valorados, entre otras razones, por la falta de transparencia en su funcionamiento, las insuficientes garantías procesales y legales, la detención de migrantes y solicitantes de asilo en instalaciones inadecuadas, el recurso a medidas represivas o el acceso insuficiente a información y asistencia legal ${ }^{95}$. Asimismo, la posición preeminente de Frontex en los hotspots, en comparación con el papel secundario que juega Easo, también ha sido reprobada, pues revela que el enfoque prioriza la identificación, el registro y el control de fronteras (Neville, et al., 2016: 37) en vez del acceso a un procedimiento de asilo justo (Rijpma, 2016: 19).

\section{LOS MANDATOS LEGALES DE FRONTEX, EASO Y EUROPOL Y LAS TAREAS OPERATIVAS DESARROLLADAS EN LA PRÁCTICA POR LAS AGENCIAS EN LOS HOTSPOTS}

Frontex, Easo y Europol interpretan de manera extensa sus mandatos legales. Sus tareas operativas en los hotspots van más allá de una mera asistencia técnica a los Estados miembros. En primer lugar, el art. 5 del Reglamento 2016/1624 de Frontex establece que «los Estados miembros serán los principa-

93 Consejo, Implementation of the counter-terrorism agenda set by the European Council, doc. 14260/16, 11.11.2016, p. 4.

94 Recomendación de la Comisión de 8.12.2016 dirigida a los Estados miembros relativa a la reanudación de los traslados a Grecia en virtud del Reglamento (UE) n. ${ }^{\circ}$ 604/2013, C(2016) 8525 final, p. 5.

95 Un análisis de las violaciones de los derechos humanos reveladas por las organizaciones no gubernamentales que trabajan en los hotspots está fuera del ámbito de estudio de este artículo. A este respecto, véanse ActionAid (2017); Amnesty International (2016); Danish Refugee Council (2017); European Union Agency for Fundamental Rights (2016); Oxfam (2016); Schalla (2015), y Majcher (2018). 
les responsables de la gestión de su sección de las fronteras exteriores» y que la «agencia apoyará la aplicación de las medidas de la Unión relacionadas con la gestión de las fronteras exteriores mediante el refuerzo, la evaluación y coordinación de las acciones de los Estados miembros para la ejecución de dichas medidas, así como en materia de retorno».

Empero, Frontex no se limita únicamente a apoyar a las autoridades nacionales en la identificación y el registro de los migrantes que llegan a los hotspots. Como se ha examinado, en la práctica Frontex asiste activamente a las autoridades nacionales en determinar la nacionalidad de los migrantes desembarcados o rescatados y ejerce una influencia decisiva en los funcionarios griegos. Estos suelen basar su decisión final sobre el país de origen de los migrantes irregulares exclusivamente en la recomendación emitida por la agencia. Las recomendaciones de Frontex respecto a la determinación de la nacionalidad de un migrante irregular presentan, por tanto, un impacto nada desdeñable ya que afectan y condicionan los ulteriores procedimientos de reubicación, asilo y retorno. Por otro lado, el Reglamento 2016/1624 de Frontex tampoco establece que la agencia sea competente para registrar las huellas dactilares de los migrantes en la base de datos de Eurodac, ni que pueda realizar entrevistas destinadas a identificar y registrar a los migrantes recién llegados a los hotspots (Guild et al., 2017: 59).

Aunque Frontex expresa reiteradamente que sus tareas operativas se limitan a proporcionar apoyo a las autoridades nacionales, el Parlamento Europeo declaró, en una resolución reciente, que "[...] la actividad de coordinación de Frontex no puede disociarse en la práctica de la actividad llevada a cabo por los Estados miembros bajo su coordinación, de modo que Frontex (y por lo tanto la UE a través de ella) podría también tener un impacto directo o indirecto en los derechos de las personas y desencadenar, como mínimo, la responsabilidad extracontractual de la UE» ${ }^{96}$. La resolución continúa diciendo que Frontex no puede eludir su responsabilidad y control «por el mero hecho de que existan acuerdos administrativos con los Estados miembros que participan en una acción coordinada por Frontex cuando dichos acuerdos repercuten en los derechos fundamentales ${ }^{97}$. Ciertamente, a pesar de que las autoridades nacionales en los hotspots ostenten en exclusividad tareas ejecutivas y decisorias, la substancial asistencia operativa que la agencia proporciona

96 Resolución del Parlamento Europeo, de 2 de diciembre de 2015, sobre el informe especial del Defensor del Pueblo Europeo relativo a su investigación de oficio OI/5/2012/BEH-MHZ sobre Frontex, (2014/2215(INI)), apdo. C.

97 Ibid., apdo. C. 
en los hotspots debería verse detallada en un instrumento legal y estar sujeta a control.

En segundo lugar, el art. 4.1, apdo. c del Reglamento 2016/794 de Europol se limita a señalar que la agencia tiene como cometido «coordinar, organizar y ejecutar cualesquiera actuaciones de investigación y operativas para respaldar y reforzar las actuaciones que lleven a cabo las autoridades competentes de los Estados miembros». Sin embargo, mientras que la misión principal de Europol en los hotspots consiste en facilitar el intercambio de información entre las autoridades nacionales, la agencia también puede desplegar agentes invitados sobre el terreno, llevar a cabo controles de seguridad, informar a los migrantes que llegan a los hotspots $\mathrm{y}$, a través del EMSC, apoyar operativamente a las autoridades nacionales en sus investigaciones sobre trata y tráfico ilegal de migrantes.

La asistencia operativa de Europol en los hotspots va desde la mera coordinación e intercambio de información hasta la recomendación a las autoridades nacionales sobre la mejor línea de acción a seguir de cara a combatir eficazmente las redes ilegales de trata y tráfico. A pesar del papel operativo que se reconoce a la agencia, el Reglamento recientemente adoptado de Europol no contiene una sola mención a las tareas de Europol en los hotspots, lo que dificulta, cuando no impide, analizar las implicaciones y el alcance real del apoyo de la agencia sobre el terreno.

Por último, si bien en los hotspots italianos las tareas de Easo se han centrado en informar sobre los procedimientos de asilo y reubicación y en facilitar la tramitación de las solicitudes de asilo, en los hotspots griegos, desde la adopción de la Declaración de la UE con Turquía y la Ley griega n. ${ }^{\circ}$ 4375/2016, la agencia también se encarga de registrar a los solicitantes de asilo y entrevistarles. Los expertos de Easo desplegados en los hotspots griegos tienen encomendada la tarea de evaluar las solicitudes de asilo y asistir al Servicio de Asilo griego en la decisión de primera instancia que ha de adoptar aceptando o rechazando la solicitud de admisibilidad presentada (European Union Agency for Fundamental Rights, 2016: 17). Asimismo, Easo apoya a las autoridades helenas en la identificación de aquellos solicitantes de asilo vulnerables ${ }^{98}$ y en el examen de las decisiones de primera instancia recurridas ante los comités griegos de apelación ${ }^{99}$. Estas tareas que Easo desarrolla en los hotspots griegos exceden manifiestamente su mandato. En concreto, el decimocuarto considerando del Reglamento 439/2010 de Easo indica que la agencia «no debe tener competencia alguna, directa ni indirecta, respecto de

98 Easo, Operating Plan Agreed by Easo and Greece, 13.12.2017, p. 13.

99

Ibid., p. 17. 
las decisiones que las autoridades de asilo de los Estados miembros adopten sobre solicitudes concretas de protección internacional».

Por tanto, desde que los hotspots fueron creados, Easo ha venido examinando el fondo de las solicitudes de asilo sin una base jurídica para ello. Los expertos desplegados por la agencia en Grecia operan en un limbo legal que difumina su específica responsabilidad y a los que no queda claro si las garantías procesales de la legislación griega les son aplicables. Precisamente, numerosas organizaciones no gubernamentales que trabajan en los hotspots señalan que los expertos de Easo ignoran ciertas garantías procesales tales como el derecho a que los solicitantes de asilo estén asistidos por un abogado mientras la agencia les entrevista (European Council for Refugees and Exiles, 2016a: 38). A este respecto, el Centro Europeo de Derechos Constitucionales y Humanos presentó en abril de 2017 una queja ante el Defensor del Pueblo Europeo arguyendo que las entrevistas llevadas a cabo por Easo en los hotspots griegos no tienen en cuenta las particularidades de cada caso y la vulnerabilidad de los solicitantes de asilo, así como que la participación de la agencia en el proceso de admisibilidad de las solicitudes de protección internacional es contraria al Reglamento 439/2010 (European Centre for Constitutional and Human Rights, 2017) ${ }^{100}$.

Además, los funcionarios de asilo griegos son, en principio, los únicos responsables a la hora de identificar a aquellos solicitantes de asilo vulnerables, a quienes no les es aplicable el procedimiento de protección internacional abreviado de los hotspots (art. 60.4. de la Ley griega n. ${ }^{\circ} 4375$ de 3 de abril de 2016). No obstante y dado el colapso del sistema de asilo griego, Easo también se ha venido encargando de identificar casos vulnerables durante sus entrevistas y de remitirlos a la oficina de asilo helena para que esta finalmente decida si se está o no ante un solicitante de asilo vulnerable ${ }^{101}$. La valoración de si existe vulnerabilidad por parte de Easo no es trivial. Si un experto desplegado por Easo no identifica o clasifica erróneamente a un solicitante como no vulnerable, su caso seguirá el procedimiento de frontera abreviado que se aplica por defecto en los hotspots griegos y que ofrece menos garantías pues ha de completarse en quince días incluyendo la fase de apelación. A este respecto, el relator especial de las Naciones Unidas sobre los derechos humanos de los migrantes mostró su preocupación en relación al procedimiento abreviado y

100 Véase Defensor del Pueblo Europeo, EASO's involvement in applications for international protection submitted in the 'hotspots' in Greece, caso: 735/2017/MDC, 13.07.2017.

101 Asylum Information Database, The concept of vulnerability in European asylum procedures, 31.07.2017, p. 30. Disponible en http://bit.ly/2f9gOmN. 
la posibilidad de que los solicitantes de asilo no estén recibiendo una audiencia justa e individualizada de sus $\operatorname{casos}^{102}$.

Las tareas que Easo desempeña respecto a la evaluación de vulnerabilidad de los solicitantes de asilo exceden la mera asistencia operativa que su reglamento le confiere. Los expertos desplegados por Easo en los hotspots gozan de un amplio margen de discrecionalidad de cara a determinar tanto si existe o no vulnerabilidad, y si una solicitud debe remitirse a las autoridades helenas para que decidan sobre la vulnerabilidad inicialmente detectada por la agencia. Incluso la decisión que adopte el sistema de asilo griego sobre la existencia o no de vulnerabilidad en un determinado caso depende en gran medida de la entrevista que previamente Easo haya realizado.

En definitiva, el enfoque de los hotspots pone de relieve que Frontex, Easo y Europol están expandiendo cada vez más sus funciones operativas a áreas en las que los Estados miembros solían presentar una competencia exclusiva. Ahora bien, el problema no radica tanto en el refuerzo de las funciones operativas de las agencias sobre el terreno como en la falta de disposiciones claras y específicas que regulen las tareas que las agencias llevan a cabo en la práctica y la asignación precisa de tareas y responsabilidades entre los numerosos actores que operan en los hotspots ${ }^{103}$. A pesar de que se haga referencia a los hotspots en el Reglamento 2016/1624 de Frontex y en la propuesta del futuro reglamento de la AEA, lo que a su vez revela que este enfoque constituye una medida a largo plazo para hacer frente a presiones migratorias extraordinarias en la UE, hasta la fecha no existe un marco legal en el que se detallen el funcionamiento, las tareas y la responsabilidad de cada agencia y autoridades nacionales en los hotspots (Neville et al., 2016: 31).

\section{LA DOCTRINA DEL TJUE DE NO DELEGACIÓN COMO LÍMITE A LA EXPANSIÓN DE LA COOPERACIÓN MULTILATERAL ENTRE FRONTEX, EASO Y EUROPOL}

Las importantes tareas operativas que Frontex, Easo y Europol llevan a cabo en los hotspots difícilmente pueden ser catalogadas como ajustadas a

102 Consejo de Derechos Humanos, Report of the Special Rapporteur on the human rights of migrants on his mission to Greece, A/HRC/35/25/Add.2, 24.04.2017, apdo. 82.

103 La asignación de responsabilidades en escenarios donde operan simultáneamente varios actores va más allá del alcance de este trabajo centrado en analizar la evolución de las tareas operativas y la cooperación de Frontex, Easo y Europol en los hotspots. A este respecto, véanse Casolari (2016: 109-134); Fernández (2017: 238-271); Fernández Rojo (2016: 105-121); Fink (2017), y Mungianu (2016). 
derecho si se aplica de manera estricta la doctrina de no delegación del TJUE. Hace sesenta años, el Tribunal de Justicia en el asunto Meroni determinó que solo podían delegarse poderes ejecutivos claramente definidos y siempre sujetos a una estricta supervisión ${ }^{104}$. Desde entonces, esta sentencia se ha esgrimido como límite a que las agencias de la UE realicen tareas que impliquen poderes legislativos, de ejecución o adjudicación ${ }^{105}$.

Recientemente, el TJUE actualizó su doctrina de no delegación y arrojó luz sobre la constitucionalidad de las agencias de la UE desde la entrada en vigor del Tratado de Lisboa, detallando en qué grado estas pueden tomar decisiones y asistir a las instituciones de la UE y a los Estados miembros en la implementación de las políticas comunitarias. En concreto, en el asunto Reino Unido c. Parlamento y Consejo de 22 de enero de 2014 se preguntó al TJUE acerca de las extensas tareas de regulación y supervisión delegadas a la Autoridad Europea de Valores y Mercados (AEVM) y su compatibilidad con la doctrina de no delegación ${ }^{106}$. El TJUE concluyó que las tareas delegadas a la AEVM no podían considerarse contrarias a derecho ya que estas se hallan clara y restrictivamente delimitadas en su reglamento constitutivo. Por tanto, el TJUE ha suavizado su inicial doctrina de no delegación al no limitar esta «a facultades de ejecución, exactamente definidas, y totalmente controladas en el uso que se hace de ellas» ${ }^{107}$, sino a tareas que "están delimitadas con precisión y pueden ser objeto de control judicial a la luz de los objetivos fijados por la autoridad delegante» ${ }^{108}$. Esto es, solo aquellas tareas delegadas a las agencias de la UE de forma imprecisa y vaga contravienen la recientemente actualizada doctrina de no delegación del TJUE.

${ }^{104}$ La Sentencia de 13 de junio de 1958, Meroni/Haute autorité, 9/56, EU:C:1958:7 sigue vigente $\mathrm{y}$ ha sido aplicado reiteradamente por el TJUE. Véanse las sentencias de 26 de mayo de 2005, Tralli/BCE, C-301/02, EU:C:2005:306; de 12 de julio de 2005, Alliance for Natural Health y otros, C-154/04, EU:C:2005:449; de 22 de enero de 2014, Reino Unido/Parlamento y Consejo, 270/12, EU:C:2014:18.

105 Véanse Chamon (2011: 1055-1075); Griller y Orator (2010: 3-35); Schneider (2009: 29-44); Scholten y Van Rijsbergen (2014: 389-405), y Van Cleynenbreugel (2014: 64-88).

106 Véanse los arts. 10.1, 17 y 18 del Reglamento (UE) 1095/2010 del Parlamento Europeo y del Consejo de 24 de noviembre de 2010 por el que se crea una Autoridad Europea de Supervisión (DO L 331 de 15.12.2010). Véanse también Adamski (2014: 819-825) y Schammo (2011: 1879-1914).

107 Sentencia de 13 de junio de 1958, Meroni/Haute autorité, 9/56, EU:C:1958:7, apdo. 150 .

108 Sentencia del Tribunal de Justicia de 22 de enero de 2014, Reino Unido/Parlamento y Consejo, 270/12, EU:C:2014:18, apdo. 53. 
El TJUE, en el asunto Reino Unido c. Parlamento y Consejo, no entró a valorar si las tareas encomendadas a la AEVM le habían sido "delegadas» o «conferidas». Esta distinción, especialmente en relación a agencias como Frontex, Easo y Europol, no es baladí, pues los principales destinatarios de su asistencia operativa y cooperación son las Administraciones nacionales y no las instituciones de la UE. A Frontex, Easo y Europol no les han sido horizontalmente «delegadas» tareas operativas pertenecientes a las instituciones de la UE, sino que sus funciones han sido verticalmente «conferidas» por los Estados miembros y la doctrina de no delegación del TJUE no les sería aplicable (Dehousse, 2002: 13; Hofmann y Morini, 2012: p. 22).

Sin embargo, aunque la atribución de tareas operativas a Frontex, Easo y Europol por parte de los Estados miembros no se sujetara a las restricciones establecidas en el asunto Meroni, ya que estas se aplican exclusivamente en supuestos de delegación, la posición del abogado general Jääskinen en el asunto Reino Unido c. Parlamento y Consejo es aquí seguida. Jääskinen consideró que el principio establecido en el asunto Meroni relativo a la prohibición de delegar competencias desmesuradamente amplias o arbitrarias sigue siendo aplicable para evaluar la legalidad de las tareas que se confieran a las agencias ${ }^{109}$. Esto es, la doctrina de no delegación en relación a Frontex, Easo y Europol es útil en tanto en cuanto los Estados miembros no podrían otorgarles tareas de aplicación o coercitivas en los hotspots que no sean lo suficientemente precisas y limitadas legalmente.

Por ello, las condiciones establecidas en el art. 28 del Reglamento 236/2012 de la AEVM son de particular interés para nuestro estudio acerca de las tareas operativas que Frontex, Easo y Europol desarrollan en los hotspots. Ello se debe a que el TJUE consideró que el art. 28 del Reglamento 236/2012 de la AEVM limitaba de manera efectiva las medidas que la agencia puede adoptar en la práctica y que, por ende, respeta la doctrina de no delegación. El art. 28.2 del Reglamento 236/2012 subraya que las medidas que la AEVM puede adoptar presentan un carácter subsidiario, pues la agencia únicamente adoptará una decisión si «[...] las autoridades competentes no han tomado medidas para hacer frente a la amenaza o las medidas adoptadas no constituyen una respuesta adecuada frente a la misma» ${ }^{10}$. En una línea similar, el art. 28.3 del Reglamento 236/2012 indica que «la AEVM tendrá en cuenta hasta qué punto la medida [...] ataja de forma significativa la amenaza que pesa sobre el correcto funcionamiento y la integridad de los mercados financieros [...]

109 Conclusiones del abogado general Jääskinen, Reino Unido/Parlamento y Consejo, 270/12, EU:C:2013:562, punto 92.

110 Ibid., apdo. 46. 
o mejora de forma significativa la capacidad de las autoridades competentes de controlar la amenaza $[\ldots] »^{111}$.

El reto radica en determinar el grado de discreción que Frontex, Easo y Europol disfrutan en la práctica en los hotspots. En nuestra opinión, la influencia que Frontex ejerce sobre los funcionarios griegos de cara a determinar la nacionalidad de los migrantes irregulares recién llegados, el apoyo operativo de Europol a las autoridades nacionales para desmantelar las redes de tráfico y trata, y la evaluación de admisibilidad de las solicitudes de asilo que lleva a cabo Easo conllevan que estas agencias del ELSJ desarrollen de facto tareas que implican una amplia facultad discrecional y que en cierta medida sean partícipes en la ejecución de una auténtica política migratoria, de asilo y de gestión fronteriza (Vos, 2018: 32).

Hasta que el TJUE conozca de un caso relativo a la constitucionalidad de las tareas operativas que Frontex, Easo y Europol ejercen en los hotspots, entendemos que estas agencias deberían estar sujetas a una interpretación más estricta de la doctrina de no delegación que la aplicada recientemente por el TJUE en el asunto Reino Unido c. Parlamento y Consejo. Frontex, Easo y Europol, a diferencia de la AEVM, son agencias que desarrollan tareas operativas en materias muy sensibles tanto para la soberanía nacional de los Estados miembros como para los derechos fundamentales de los migrantes a los que asisten. Ello justifica una interpretación más restringida por parte del TJUE de su doctrina de no delegación.

A este respecto, el TJUE en el asunto Parlamento c. Consejo de 5 de septiembre de 2012 relativo a la anulación de la Decisión 2010/252/UE del Consejo, que complementaba el Código de Fronteras Schengen respecto a la vigilancia marítima en operaciones coordinadas por Frontex, expuso que las tareas conferidas a Frontex presentan una naturaleza distintiva ${ }^{12}$. El TJUE destacó la especial naturaleza de las materias del ELSJ en comparación con otros ámbitos competenciales de la UE. En particular, el Tribunal consideró que la adopción de normas sobre la atribución de tareas de ejecución a los guardias fronterizos debe ser un aspecto reservado exclusivamente al legislador de la UE, pues ello puede afectar a los derechos fundamentales de las personas

111 Ibid., apdo. 47.

112 Sentencia del Tribunal de Justicia de 5 de septiembre de 2012, Parlamento/Consejo, 355/10, EU:C:2012:516. Véanse Acosta y González (2014: 267-284); Chamon (2013: 849-860), y Den Heijer y Tauschinsky (2013: 513-533). Y también la Sentencia de 10 de septiembre de 2015, Parlamento/Consejo, C-363/14, EU:C:2015:579. 
y se hace necesario adoptar decisiones políticas ${ }^{113}$. El abogado general Mengozzi también indicó que «[...] los límites de las tareas de ejecución deben definirse, sobre todo, por referencia a las características de la política de que se trate» ${ }^{114}$.

En definitiva, si bien una delegación incondicionada de funciones a Frontex, Easo o Europol en los hotspots es contraria a derecho dada la insistencia del TJUE en que las tareas de la AEVM presenten una naturaleza subsidiaria, conferir a Frontex, Easo o Europol tareas operativas estrictamente delimitadas puede considerarse conforme con la doctrina de no delegación del TJUE. Sin embargo, como hemos estudiado, las tareas de Frontex, Easo o Europol en los hotspots no se circunscriben a la mera prestación de asistencia técnica a los Estados miembros, sino que estas desarrollan una verdadera cooperación operativa no condicionada de manera restrictiva en ningún marco legal a nivel nacional o de la UE, lo que difícilmente tiene un encaje incluso con la recientemente suavizada doctrina de no delegación del TJUE.

\section{v. CONCLUSIÓN}

En los hotspots, los Estados miembros ya no son exclusivamente competentes para registrar, identificar, informar, retornar a los migrantes irregulares, examinar sus solicitudes de asilo o investigar las redes de trata y tráfico ilegal de migrantes. Las autoridades nacionales que se enfrenten a una presión migratoria extraordinaria en sus fronteras exteriores y soliciten la creación de hotspots en su territorio deben cooperar con Frontex, Easo y Europol. Mientras que la cooperación entre estas agencias ha sido una constante desde su creación, el enfoque de los hotspots introducido por la Agenda Europea de la Migración en 2015 como resultado de la "crisis de los refugiados» ha reforzado e institucionalizado la cooperación operativa y multilateral entre estas agencias.

La complementariedad de las tareas de Frontex, Easo y Europol y la creciente necesidad de proporcionar una asistencia operativa coordinada a los Estados miembros más afectados por la llegada masiva de flujos migratorios mixtos a sus costas fueron factores clave en la introducción de los hotspots. Este enfoque tiene como objetivo promover el establecimiento de sinergias entre las agencias, evitar la duplicación y la superposición de sus tareas, y perfeccionar

113 Sentencia de 5 de septiembre de 2012, Parlamento/Consejo, 355/10, EU:C:2012:516, apdos. 76 y 77.

114 Conclusiones del abogado general Mengozzi, Parlamento/Consejo, 355/10, EU:C: 2012:207, punto 29. 
la coordinación operativa sobre el terreno con las autoridades nacionales y locales. Formalmente, el enfoque de los hotspots no confiere a Frontex, Easo o Europol ningún poder ejecutivo, de decisión o coercitivo, lo que sería contrario a los tratados, a los reglamentos de las agencias y a la doctrina del TJUE de no delegación. Sin embargo, las tareas operativas y la cooperación de estas agencias sobre el terreno presentan de facto un claro impacto en la implementación de las políticas y leyes adoptadas a nivel europeo en materia de gestión fronteriza, migración y asilo.

El ELSJ es un área caracterizada por su naturaleza operativa que precisa necesariamente para su efectividad de una suficiente capacidad administrativa sobre el terreno. Por ello, el papel de Frontex, Easo y Europol en los hotspots se centra en asistir operativamente a las autoridades nacionales en la aplicación efectiva y uniforme de la política y legislación adoptada por la UE. Si bien las tareas operativas de estas agencias son y seguirán siendo cruciales para garantizar respuestas consistentes y coherentes en situaciones de crisis y emergencia en las fronteras exteriores de la Unión, el cometido de implementación de estas agencias ha de venir necesariamente acompañado de un instrumento legal que regule y detalle sus tareas y su cooperación multilateral. Este instrumento legislativo no solo aclararía la asignación de responsabilidades entre los diversos actores que cooperan estrechamente en los hotspots, sino que también proporcionaría cierta transparencia con respecto al funcionamiento de los mismos.

\section{Bibliografía}

ACNUR. (2016). Building on the Lessons Learned to Make the Relocation Schemes Work More Effectively. Disponible en: https://bit.ly/2zPrqC4.

Acosta Sánchez, M. A. y González García, I. (2014). TJUE. Sentencia de 05-092012, Parlamento c. Consejo, C-355/10: Vigilancia de fronteras marítimas y elementos esenciales en los actos de ejecución. Revista de Derecho Comunitario Europeo, 47, 267-284.

ActionAid. (2017). Transitioning to a Government-Run Refugee and Migrant Response in Greece. Joint Agency Briefing Paper. Disponible en: https://bit.ly/2Jgm45F.

Adamski, D. (2014). The ESMA doctrine: A constitutional revolution and the economics of delegation. European Law Review, 6, 812-834.

Amnesty International. (2016). Hotspot Italy How EU's Flagship Approach Leads to Violations of Refugee and Migrant Rights. Disponible en: https://bit.ly/2eCgnTE.

Antonakaki, M., Kasparek, B. y Maniatis, G. (2016). Counting Heads and Channeling Bodies. The Hotspot Centre Vial in Chios, Greece. Transit Migration, 2. Disponible en: https://bit.ly/2tV9OUm. 
Argomaniz, J. (2012). A “coordination nightmare”? Institutional coherence in European Union counter-terrorism. En C. Kaunert, S. Léonard y P. Pawlak, Patryk (eds.). European Homeland Security: A European Strategy in the Making? (pp. 90-112). London: Routledge.

Basilien-Gainche, M. L. (2017). Hotspots, Cold Facts. Managing Migration by Selecting Migrants. En C. Grutters, S. Mantu y P. Minderhoud (eds.). Migration on the Move: Essays on the Dynamics of Migration (pp. 153-171). Leiden: Brill.

Blasi Casagran, C. (2016). El reglamento europeo de Europol: Un nuevo marco jurídico para el intercambio de datos policiales en la UE. Revista General de Derecho Europeo, 40, 202-221.

- (2018). El papel de Europol en la lucha contra el tráfico de migrantes y la trata de seres humanos. Revista de Derecho Comunitario Europeo, 59, 333-357. Disponible en: https://doi.org/10.18042/cepc/rdce.59.09.

Brady, H. (2008). Europol and the European criminal intelligence model: a non-state response to organized crime. Policing: A Journal of Policy and Practice, 2 (1), 103-109. Disponible en: https://doi.org/10.1093/police/pan014.

Busuioc, M. (2016). Friend or Foe? Inter-Agency Cooperation, Organizational Reputation, and Turf. Public Administration, 94(1), 40-56. Disponible en: https:// doi.org/10.1111/padm.12160.

Campesi, G. (2018). European Border and Coast Guard (Frontex): Security, Democracy, and Rights at the EU Border. Oxford Research Encyclopedia of Criminology, 1-32. Disponible en: https://doi.org/10.1093/acrefo$\mathrm{re} / 9780190264079.013 .354$.

Carrera, S., Blockmans, S., Cassarino, J. P., Gros, D., Guild, E. y Letta, E. (2017). The European Border and Coast Guard Addressing migration and asylum challenges in the Mediterranean? CEPS Task Force Report. Disponible en: http://aei. pitt.edu/83997/.

Carrera, S. y Den Hertog, L. (2016). A European Border and Coast Guard: What's in a Name? CEPS Paper in Liberty and Security in Europe, 88, 1-22. Disponible en: https://bit.ly/2zO3wqh.

Casolari, F. (2016). The EU's hotspot approach to managing the migration crisis: a blind spot for international responsibility? The Italian Yearbook of International Law Online, 25 (1), 109-134. Disponible en: https://doi. org/10.1163/22116133-90000109.

Chamon, M. (2011). EU agencies between Meroni and Romano or the devil and the deep blue sea. Common Market Law Review, 48, 1055-1075.

(2013). How the Concept of Essential Elements of a Legislative Act Continues to Elude the Court, Parliament v. Council. Common Market Law Review, 50, 849-860.

Christiansen, T. (2001). Intra-institutional politics and inter-institutional relations in the EU: towards coherent governance? Journal of European Public Policy, 8 (5), 747-769. Disponible en: https://doi.org/10.1080/13501760110083491.

Danish Refugee Council. (2017). Fundamental Rights and the EU Hotspot Approach. Disponible en https://bit.ly/2Sk7WMK. 
De Bruycker, P. (2016). The European border and coast guard: a new model built on an old logic. European papers: a journal on law and integration, 1(2), 559-569. Disponible en: https://doi.org/10.15166/2499-8249/53.

Dehousse, R. (2002). Misfits: EU law and the transformation of European governance. Jean Monnet Working Paper, 2.

Del Valle Gálvez, A. (2016). Los refugiados, las fronteras exteriores, y la evolución del concepto de frontera internacional. Revista de Derecho Comunitario, 55, 759777.

Den Heijer, M. y Tauschinsky, E. (2013). Where Human Rights Meet Administrative Law: Essential Elements and Limits to Delegation. European Constitutional Law Review, 9 (3), 513-533.

Den Heijer, M., Rijpma, J. y Spijkerboer, T. (2016). Coercion, prohibition, and great expectations: The continuing failure of the Common European Asylum System. Common Market Law Review, 53 (3), 607-642.

Donaire Villa, F. J. (2015). ¿De qué hablamos cuando hablamos de coordinación en el ámbito de la Unión Europea, y por tanto, del ELSJ? En F. J. Donaire Villa y A. Olesti Rayo (eds.). Técnicas y ámbitos de coordinación en el Espacio de Libertad, Seguridad y Justicia (pp. 39-46). Madrid: Marcial Pons.

European Centre for Constitutional and Human Rights. (2017). EASO's influence on inadmissibility decisions exceeds the agency's competence and disregards fundamental rights. Disponible en: https://bit.ly/2yC7eCt.

European Council for Refugees and Exiles (ECRE). (2016a). The implementation of the hotspots in Italy and Greece. Disponible en: https://bit.ly/2s0I37g. (2016b). With Greece: Recommendations for refugee protection. Disponible en: https://bit.ly/2PsDYId.

European Union Agency for Fundamental Rights. (2016). Opinion of the European Union Agency for Fundamental Rights on fundamental rights in the "hotspots" set up in Greece and Italy. Opinion 5/2016.

Fernández, M. (2017). Multi-Stakeholder Operations of Border Control Coordinated at the EU Level and the Allocation of International Responsibilities. En T. Gammeltoft-Hansen y J. Vedsted-Hansen (eds.). Human Rights and the Dark Side of Globalisation (pp. 238-271). London: Routledge.

Fernández Rojo, D. (2016). Hotspot approach: cooperación y control del Frontex, Easo y Europol. En C. Blasi Casagran y M. Illamola Dausà (eds.). El control de las agencias del Espacio de Libertad, Seguridad y Justicia (pp. 105-123). Madrid: Marcial Pons.

(2017). Reglamento 2016/1624: De Frontex a la guardia europea de fronteras y costas. Revista General de Derecho Europeo, 41, 223-251.

Ferraro, F. y De Capitani, E. (2016). The new European Border and Coast Guard: yet another «half way" EU reform? ERA Forum, 17 (3), 385-398. Disponible en: https://doi.org/10.1007/s12027-016-0447-y.

Fink, M. (2017). Frontex and Human Rights: Responsibility in "Multi-Actor Situations" under the ECHR and EU Public Liability Law [tesis doctoral inédita]. Leiden University; University of Vienna. 
Franco-García, M. Á. (2018). El alcance de la cooperación entre las agencias de la Unión Europea implicadas en la seguridad. Revista General de Derecho Europeo, 44, 13-54.

Gkliati, M. (2017). The Application of the EU-Turkey Agreement: A Critical Analysis of the Decisions of the Greek Appeals Committee. European Journal of Legal Studies, 10 (1), 81-123.

Goodwin-Gill, G. S. (2015). Refugees and Migrants at Sea: Duties of Care and Protection in the Mediterranean and the Need for International Action. JMCE Migrants. Disponible en: https://bit.ly/2ArGOov.

Griller, S. y Orator, A. (2010). Everything under control? The "way forward" for European agencies in the footsteps of the Meroni doctrine. European Law Review, 35 (1), 3-35.

Guild, E. (2016). Does the EU Need a European Migration and Protection Agency? International Journal of Refugee Law, 28 (4), 585-600. Disponible en: https:// doi.org/10.1093/ijrl/eew038.

Guild, E., Costello, C. y Moreno-Lax, V. (2017). Implementation of the 2015 Council Decisions establishing provisional measures in the area of international protection for the benefit of Italy and of Greece. Study for the LIBE Committee of the European Parliament, PE 583132.

Hertog, L. y Strob, S. (2013). Coherence in EU External Relations: Concepts and Legal Rotting of an Ambiguous Term. European Foreign Affairs Review, 18 (3), 373-388.

Hofmann, H. C., Morini, A. (2012). Constitutional Aspects of the Pluralisation of the EU Executive through "Agencification". University of Luxembourg Law Working Paper Series, 1.

Horii, S. (2018). Accountability, Dependency, and EU Agencies: The Hotspot Approach in the Refugee Crisis. Refugee Survey Quarterly, 0, 1-27. Disponible en: https://doi.org/10.1093/rsq/hdy005.

Majcher, I. (2018). The EU Hotspot Approach: Blurred Lines between Restriction on and Deprivation of Liberty. Border Criminologies [blog], 6-04-2018. Disponible en: https://bit.ly/2qbjiq0.

Moreno-Lax, V. (2015). Europe in Crisis: Facilitating Access to Protection (Discarding) Offshore Processing and Mapping Alternatives for the Way Forward. Red Cross EU Office. Disponible en: https://bit.ly/2HgQRB2.

Mungianu, R. (2016). Frontex and non-refoulement: the international responsibility of the EU. Cambridge: Cambridge University Press.

Neville, D., Sy, S. y Rigon, A. (2016). On the frontline: The hotspot approach to managing migration. Study for the LIBE Committee of the European Parliament, PE 556.942.

Oxfam. (2016). Hotspot, Rights Denied. Oxfam Briefing Paper. Disponible en: https:// bit.ly/2IUBtb0.

Papageorgiou, I. (2017). International protection in Greece. Background information for the LIBE Committee delegation to Greece 22-25 May 2017. Study for the LIBE Committee of the European Parliament, PE 583.145. 
Pérez González, C. (2016). De Frontex a la Agencia Europea de la Guardia Europea de Fronteras y Costas: ¿cambia todo para que todo siga igual? A propósito de la necesidad de mejorar la protección de los derechos fundamentales. En C. Blasi Casagran y M. Illamola Dausà (eds.). El control de las agencias del Espacio de Libertad, Seguridad y Justicia (pp. 192-208). Madrid: Marcial Pons.

Pi Llorens, M. (2017). El nuevo mapa de las agencias europeas del Espacio de Libertad, Seguridad y Justicia. Revista de Derecho Comunitario Europeo, 56, 77-117.

Rijpma, J. J. (2016). The Proposal for a European Border and Coast Guard: Evolution or Revolution in External Border Management? Study for the LIBE Committee of the European Parliament, PE 556.934.

Rodier, C. (2017). Le faux semblant des hotspots. La Revue des Droits de l'Homme, 13, 1-22. Disponible en: https://doi.org/10.4000/revdh.3375.

Santos Vara, J. (2018). La transformación de Frontex en la Agencia Europea de la Guardia de Fronteras y Costas: ¿hacia una centralización en la gestión de las fronteras? Revista de Derecho Comunitario Europeo, 59, 143-186. Disponible en: https://doi.org/10.18042/cepc/rdce.59.04.

Schalla, M. (2015). Observations at the Campsite of Moria on Lesvos, October $22^{\text {nd }}$ and $24^{\text {th }}$, 2015. Disponible en: http://bit.ly/2ryrC6s.

Schammo, P. (2011). The European Securities and Markets Authority: Lifting the Veil on the Allocation of Powers. Common Market Law Review, 48, 1879-1914.

Schneider, J. P. (2009). A Common Framework for Decentralized EU Agencies and the Meroni Doctrine. Administrative Law Review, 61, 29-44.

Scholten, M. y Van Rijsbergen, M. (2014). The ESMA-short selling case: Erecting a new delegation doctrine in the EU upon the Meroni-Romano remnants. Legal Issues of Economic Integration, 41(4), 389-405.

Scipioni, M. (2017). De Novo Bodies and EU Integration: What is the Story behind EU Agencies' Expansion? Journal of Common Market Studies, 1-17. Disponible en: https://doi.org/10.1111/jcms.12615.

Soler García, C. (2017). La guardia europea de fronteras y costas: ¿Un avance respecto a Frontex? Una valoración provisional. Revista Electrónica de Estudios Internacionales (REEI), 34 (4), 1-44. Disponible en: https://doi.org/10.17103/reei.34.05.

Thym, D. (2016). The "refugee crisis" as a challenge of legal design and institutional legitimacy. Common Market Law Review, 53 (6), 1545-1573.

Triandafyllidou, A. y Mantanika, R. (2017). Emergencia de refugiados en el Mediterráneo: evaluación de las respuestas políticas de la Unión Europea. Migración y Desarrollo, 15 (28), 7-38.

Tribunal de Cuentas Europeo (2017). Respuesta de la UE a la crisis de los refugiados: el enfoque de «puntos críticos». Disponible en: https://bit.ly/2zbnjib.

Tsourdi, E. (2016). Bottom-up salvation? From practical cooperation towards joint implementation through the European Asylum Support Office. European Papers, 1, 997-1031. Disponible en: https://doi.org/10.15166/2499-8249/115. (2017a). Solidarity at work? The prevalence of emergency-driven solidarity in the administrative governance of the Common European Asylum System. 
Maastricht Journal of European and Comparative Law, 24 (5), 1-20. Disponible en: https://doi.org/10.1177/1023263X17742801.

(2017b). Hotspots and EU Agencies: Towards an Integrated European Administration. EU Immigration and Asylum Law and Policy [blog], 26-01-2017. Disponible en: http://eumigrationlawblog.eu/hotspots-and-eu/.

Van Cleynenbreugel, P. (2014). Meroni circumvented? Article 114 TFEU and EU regulatory agencies. Maastricht Journal of European and Comparative Law, 21 (1), 64-88. Disponible en: https://doi.org/10.1177/1023263X1402100104.

Vos, E. (2018). EU agencies on the move: challenges ahead. Swedish Institute for European Policy Studies, 1.

Weyemberg, A., Armada, I. y Brière, C. (2014). The inter-agency cooperation and future architecture of the EU criminal justice and law enforcement area. Study for the LIBE Committee, PE 510.000. 